\title{
Classification of Stable Symmetric Markov Chains
}

\section{MARTIN L. SILVERSTEIN}

\author{
Communicated by M. RosenblatT
}

Introduction. We study standard transition matrices $\boldsymbol{P}_{t}(x, y)$ defined for $x, y$ in a denumerably infinite state space $I$ and satisfying two conditions:

0.1. Every state is stable. That is, for every $x$ in $I$

$$
q(x)=\operatorname{Lim}(1 / t)\left\{1-\boldsymbol{P}_{t}(x, x)\right\} \quad(t \downarrow 0)
$$

(which always exists in the extended sense) is finite.

0.2. There exists a symmetrizing speed measure $m$. That is

$$
m(x) \boldsymbol{P}_{t}(x, y)=m(y) \boldsymbol{P}_{t}(y, x)
$$

for $t>0$ and $x, y$ in $I$.

We do not assume that $\boldsymbol{P}_{t}$ is conservative.

Under these conditions there exists a substochastic matrix $P$ such that

$$
q(x) P(x, y)=\operatorname{Lim}(1 / t) \boldsymbol{P}_{t}(x, y) \quad(r \downarrow 0)
$$

for $x \neq y$ in $I$. It will turn out that there is no loss of generality in assuming that $P$ is irreducible and transient and we do so from now on.

Following W. Feller [7] we refer to $m$ as the speed measure and to $P$ as the road map. For fixed $m$ and $P$ we classify all standard transition matrices $\boldsymbol{P}_{\boldsymbol{t}}$ satisfying (0.1), (0.2) and (0.3) in terms of certain Dirichlet spaces on the active part of the extremal Martin boundary for $P$. (The rate $q$ is determined up to a constant multiple since the product $m q$ must symmetrize $P$.)

To simplify the presentation we postulate that the road map $P$ is strictly stochastic. For the substochastic case the techniques of this paper are adequate only when the transition matrix $\boldsymbol{P}_{t}$ satisfies the Kolmogorov equations. We plan to treat the general case in a future publication.

Added in proof. See section 26 in [20].

Our basic tools are certain techniques introduced in [17]. In the interest of accessibility we have kept this paper as self contained as we could. In particular we have kept references to [17] to a minimum. 
We begin in Section 1 by collecting some well known results for transient discrete time Markov chains up to and including the Martin representation and the appropriate analogue of the kernel introduced by Naim [13] for Brownian motion. Our main source here is the classic paper of G. Hunt [11].

In Section 2 we study an associated discrete time Dirichlet space and in Section 3 we establish some important formulae for discrete time Dirichlet norms.

The last three Sections are devoted to continuous time. We consider in Section 4 a general standard transition matrix $\boldsymbol{P}_{t}(x, y)$ satisfying $(0.1)$ through (0.3) and establish elementary results for the corresponding Dirichlet space. Also for the reader's convenience we collect general results on Dirichlet spaces, in particular spelling out in detail the connection with symmetric submarkovian semigroups. The corresponding minimal transition matrix and Dirichlet space are studied in Section 5. Finally the desired classification is obtained in Section 6. Only here do we use results from [17] in a nontrivial way. For the reader's convenience several of the crucial arguments from [17] are reproduced with appropriate simplifications.

Our classification is analogous to the one obtained by M. Fukushima [10] for Brownian motion. Our techniques however are quite different.

We do not consider the "construction problem" for the processes themselves. However it follows from the results in [17] that a decent process on an appropriate compactification always exists and that the classifying Dirichlet space corresponds to the process on the complement of $I$ obtained via random time change.

To our knowledge a complete classification has not been obtained previously even under the restriction (0.2) without assumptions of finiteness on the boundary. For related results we refer to the paper of Elliott [6] and to the paper of Shiga and Watanabe [16]. For results without the restriction (0.2) we refer to the work of Chung [3, 4]. For background material on continuous time Markov chains we refer to Chapters 1, 2 and 3 in Part II of [2]. For background material on discrete time transient potential theory we refer to [12].

Analogous problems in a more general context are considered in [18]. Also a paper is being prepared in which the techniques used here are extended to continuous time Markov chains with instantaneous states.

Added in proof. See [21].

As in [17] we gratefully acknowledge our debt to M. Fukishima who seems to have been the first to appreciate the significance of Dirichlet spaces as a tool for studying symmetric Markov processes.

Added in proof. The main results are established and extended by quite different techniques in the forthcoming monograph [20]. Also the first chapter in [20] contains in improved form the relevant material from [17].

Notations. Functions and measures on $I$ will be viewed as column vectors and row vectors and then matrices will be viewed as operators according to the usual conventions.

The indicator of a set $A$ will be denoted both by $1_{A}$ and $I(A)$. 
The integral of a function $\xi$ over the set determined by a particular condition, say $X_{t}=y$, will be denoted both by $\varepsilon\left[X_{t}=y ; \xi\right]$ and by $\varepsilon I\left(X_{t}=y\right) \xi$.

Questions of measurability will always be taken for granted. For example there will always be an obvious candidate for the underlying $\sigma$-algebra and all functions are assumed measurable with respect to this $\sigma$-algebra.

All functions are real valued. In particular $L^{2}(\mu)$ or $L^{2}(X, \mu)$ is the real Hilbert space of square integrable functions on the measure space $(X, \mu)$, and $C_{\text {com }}(X)$ is the collection of real valued continuous functions on $X$ with compact support.

1. Discrete time potential theory. Throughout the paper $\boldsymbol{I}$ is a denumerably infinite set and $P(x, y)$ is a stochastic matrix on $I$ which vanishes on the diagonal. Thus

$$
P(x, y) \geqq 0 ; \quad P(x, x)=0 ; \quad \sum_{y} P(x, y)=1 .
$$

We will always be governed by the

\subsection{Basic hypothesis.}

1.1.1. There exists an everywhere positive measure $\alpha$ on $I$ such that

$$
\alpha(x) P(x, y)=\alpha(y) P(y, x) \quad x, y \text { in } I .
$$

\subsubsection{The matrix $P$ is irreducible and transient}

We will generally refer to $I$ as the state space, to $P$ as the road map and to $\alpha$ as the symmetrizing measure.

The symmetry condition (1.2) is a fundamental restriction. However 1.1.2 is natural since in the recurrent case the classification theory is trivial. (See the remark following Theorem 5.1.) Of course $\alpha$ is unique up to a constant multiple.

By transience of $P$, the potential operator

$$
N(x, y)=\sum_{n=0}^{\infty} P^{n}(x, y)
$$

is finite and by irreducibility and symmetry of $P$, every entry $N(x, y)>0$. A function $h$ on $I$ is excessive if it is finite and

$$
h \geqq 0 ; \quad h \geqq P h .
$$

From irreducibility of $P$ it follows that if $h$ is excessive and nontrivial then $h(x)>0$ for $x$ in $I$. A function $g$ on $I$ is harmonic if

$$
g=P g
$$

with the understanding that the right side converges absolutely. It is well known and trivially verified that every excessive function $h$ has a unique Riesz decomposition

$$
h=N(1-P) h+r^{h}
$$


with $r^{h}$ nonnegative and harmonic. An excessive function $h$ is called a potential if $r^{h}=0$.

We augment the state space $I$ by introducing a dead point $\partial$. Functions $f$ defined on $I$ are extended to $I \cup\{\partial\}$ via the convention

$$
f(\partial)=0 .
$$

The standard discrete time sample space $\Lambda$ is the set of sequences $\omega=\{\omega(n)\}_{n=0}^{\infty}$ in the augmented state space $I \cup\{\partial\}$ which satisfy the following condition:

1.2. There exists a lifetime $\zeta(\omega)$ with $0 \leqq \zeta(\omega) \leqq+\infty$ such that $\omega(n)=\partial$ if and only if $n \geqq \zeta(\omega)$.

The trajectory variables $X_{n}$ are defined on $\Lambda$ by

$$
X_{n}(\omega)=\omega(n) .
$$

For $x$ in $I$ the probability $\mathfrak{B}_{x}$ is the unique probability on $\Lambda$ such that

$$
\mathfrak{P}_{x}\left[X_{0}=x, X_{1}=x_{1}, \cdots, X_{n}=x_{n}\right]=P\left(x, x_{1}\right) \cdots P\left(x_{n-1}, x_{n}\right)
$$

for every choice of $n \geqq 0$ and of $x_{1}, \cdots, x_{n}$ in $I$. More generally for $h$ a nontrivial excessive function, the conditional road map $P^{h}$ is defined by

$$
P^{h}(x, y)=(1 / h(x)) P(x, y) h(y)
$$

and the conditional probability $\mathfrak{P}^{h}{ }_{x}$ on $\Lambda$ is determined by

$$
\mathfrak{P}_{x}^{h}\left[X_{0}=x, \cdots, X_{n}=x_{n}\right]=P^{h}\left(x, x_{1}\right) \cdots P^{h}\left(x_{n-1}, x_{n}\right) .
$$

In particular $h(x) \mathfrak{P}_{x}^{h}[\zeta>n]=P^{n} h(x)$.

For $E$ a subset of $I$ the hitting time $\sigma(E)$ and the delayed hitting time $\sigma^{+}(E)$ are defined by

$$
\begin{aligned}
\sigma(E) & =\inf \left\{n \geqq 0: X_{n} \text { is in } E\right\} \\
\sigma^{+}(E) & =\inf \left\{n>0: X_{n} \text { is in } E\right\}
\end{aligned}
$$

with the understanding that these times are $+\infty$ when not otherwise defined. The hitting probability $H^{E}(x, y)$ is defined by

$$
H^{E}(x, y)=\mathfrak{B}_{x}\left(\sigma(E)<+\infty ; X_{\sigma(E)}=y\right] .
$$

If $g$ is harmonic then also

$$
H^{M} g=g
$$

whenever $D=I-M$ is finite. This follows from the martingale convergence theorem [12, Chap. 3] together with the trivial estimate

$$
\sup _{n \leqq \sigma(M)}\left|g\left(X_{n}\right)\right| \leqq\left|g\left(X_{\sigma(M)}\right)\right|+\sup _{x \text { in } D}|g(x)| .
$$

The last exit time $\sigma^{*}(E)$ is defined by

$$
\sigma^{*}(E)=\sup \left\{n \geqq 0: X_{n} \text { is in } E\right\}
$$


with the understanding that $\sigma^{*}(E)=-\infty$ when not otherwise defined.

For $h>0$ excessive define

$$
\begin{aligned}
L^{h}(x) & =\mathfrak{P}_{x}^{h}[\zeta=1] \\
L_{E}^{h}(x) & =1_{E}(x) \mathfrak{P}_{x}^{h}\left[\sigma^{+}(E)=+\infty 1\right. \\
r^{h}(x) & =h(x) \mathfrak{P}_{x}^{h}[\zeta=+\infty] \\
r_{E}^{h}(x) & =h(x) \mathfrak{P}_{x}^{h}\left[\sigma(E)<+\infty ; \sigma^{+}(E)=+\infty\right]
\end{aligned}
$$

and we are ready to refine the Riesz decomposition (1.5).

Theorem 1.1. Let $h>0$ be excessive and let $E$ be a subset of $I$.

(i) $h$ has the Riesz decomposition

$$
h=N\left(h L^{h}\right)+r^{h} .
$$

(ii) $g=H^{E} h$ is excessive and has the Riesz decomposition

$$
g=N\left(h L_{E}^{h}\right)+r_{E}^{h} .
$$

(iii) $h(x) \mathfrak{P}_{x}^{h}\left[X_{n}=y\right.$; $\left.\zeta=n+1\right]=P^{n}(x, y) h(y) L^{h}(y)$

$$
\begin{aligned}
& h(x) \mathfrak{P}_{x}^{h}\left[X_{\zeta-1}=y ; \zeta<\infty\right]=N(x, y) h(y) L^{h}(y) \\
& h(x) \mathfrak{P}_{x}^{h}[\zeta<\infty]=N\left(h L^{h}\right)(x) \\
& \left.h(x) \mathfrak{P}_{x}^{h}\left[\zeta=+{ }^{i}\right]\right]=r^{h}(x) .
\end{aligned}
$$

(iv) $h(x) \mathfrak{P}_{x}^{h}\left[\sigma^{*}(E)=n ; X_{\sigma^{*}(E)}=y\right]=P^{n}(x, y) L_{E}^{h}(y) h(y)$

$$
\begin{gathered}
=g(x) \mathfrak{P}_{x}^{o}\left[X_{n}=y ; \zeta=n+1\right] \\
\begin{aligned}
h(x) \mathfrak{P}_{x}^{h}\left[0 \leqq \sigma^{*}(E)<+\infty ; X_{\sigma^{*}(E)}=y\right] & =N(x, y) L_{E}^{h}(y) h(y) \\
& =g(x) \mathfrak{P}_{x}^{o}\left[X_{\zeta-1}=y ; \zeta<+\infty\right] \\
h(x) \mathfrak{P}_{x}^{h}\left[0 \leqq \sigma^{*}(E)<+\infty\right]=N\left(L_{E}^{h} h\right)(x) & \\
=g(x) \mathfrak{P}_{x}^{o}[\zeta<+\infty] . & <+\infty]=r_{E}^{h}(x) \\
& =g(x) \mathfrak{P}_{x}^{o}[\zeta=+\infty] \\
h(x) \mathfrak{P}_{x}^{h}\left[\sigma(E)<+\infty ; \sigma^{*}(E)=+\infty\right. &
\end{aligned} \\
h(x) \mathfrak{P}_{x}^{h}[\sigma(E)=+\infty]=h(x)-g(x) .
\end{gathered}
$$

(i) follows from (iii) and (iii) is elementary if the identities are treated in the order stated. (ii) and (iv) require a bit more care but are also easy. Complete proofs can be found in [12].

The time reversal operator $\rho$ is defined on $\Lambda \cap[\zeta<+\infty]$ by

$$
\rho \omega(n)=\left\{\begin{array}{cc}
\omega(\zeta-1-n) & 0 \leqq n \leqq \zeta-1 . \\
\partial & n \geqq \zeta
\end{array}\right.
$$


For $E$ a subset of $I$ the local time reversal operator $\rho_{E}$ and the truncation operator $\tau_{E}$ are defined on $\Lambda \cap\left[\sigma(E)<+\infty ; \sigma^{*}(E)<+\infty\right]$ by

$$
\begin{aligned}
& \rho_{E} \omega(n)=\left\{\begin{array}{lr}
\omega\left[\sigma^{*}(E)-n\right] & 0 \leqq n \leqq \sigma^{*}(E) \\
\partial & n>\sigma^{*}(E)
\end{array}\right. \\
& \tau_{E} \omega(n)=\left\{\begin{array}{lr}
\omega(n) & 0 \leqq n \leqq \sigma^{*}(E) . \\
\partial & n>\sigma^{*}(E) .
\end{array}\right.
\end{aligned}
$$

The behavior of the probabilities $\mathfrak{B}_{x}^{h}$ under these operators is described in

Theorem 1.2. Let $h, h^{\prime}>0$ be excessive, let $E, E^{\prime}$ be subsets of $I$ and let $\xi \geqq 0$ be defined on $\Lambda$. Then

$$
\begin{aligned}
& \sum_{x} \alpha(x) h^{\prime}(x) L^{h^{\prime}}(x) h(x) \S_{x}^{h}[\zeta<+\infty ; \xi \circ \rho] \\
& =\sum_{\nu} \alpha(y) h(y) L^{h}(y) h^{\prime}(y) \xi_{h}^{h^{\prime}}[\zeta<+\infty ; \xi] \\
& \sum_{x} \alpha(x) h^{\prime}(x) L_{E^{\prime}}^{h^{\prime}}(x) h(x) \S_{x}^{h}\left[\sigma(E)<+\infty ; \sigma^{*}(E)<+\infty ; \xi \circ \rho_{E}\right] \\
& \quad=\sum_{y} \alpha(y) h(y) L_{E}^{h}(y) h^{\prime}(y) \xi_{y}^{h^{\prime}}\left[\sigma\left(E^{\prime}\right)<+\infty ; \sigma^{*}\left(E^{\prime}\right)<+\infty ; \xi \circ \tau_{E}\right] .
\end{aligned}
$$

To our knowledge results of this nature were first obtained by G. Hunt in [11]. It suffices to prove (1.9) and then only for the special case $\xi=\prod_{0 \leqq i \leqq n}$ $I\left(X_{i}=x_{i}\right)$ with $x_{0}$ in $E$. The left side of (1.9)

$$
\begin{aligned}
& =\sum_{x} \alpha(x) h^{\prime}(x) L_{E}^{h^{\prime}}(x) N\left(x, x_{n}\right) P\left(x_{n}, x_{n-1}\right) \cdots P\left(x_{1}, x_{0}\right) h\left(x_{0}\right) L_{E}^{h}\left(x_{0}\right) \\
& =\alpha\left(x_{0}\right) h\left(x_{0}\right) L_{E}^{h}\left(x_{0}\right) P\left(x_{0}, x_{1}\right) \cdots P\left(x_{n-1}, x_{n}\right) N\left(h^{\prime} L_{E}^{h^{\prime}}\right)\left(x_{n}\right) \\
& =\alpha\left(x_{0}\right) h\left(x_{0}\right) L_{E}^{h}\left(x_{0}\right) h^{\prime}\left(x_{0}\right) \S_{x_{0}}^{h^{\prime}}\left[\xi ; n \leqq \sigma^{*}\left(E^{\prime}\right)<+\infty\right]
\end{aligned}
$$

which is the right side of (1.9).

To make further progress we introduce an extended sample space $\Lambda_{\infty}$ and appropriate measures on $\Lambda_{\infty}$ as outlined in [11].

Let $I_{k}, k \geqq 1$ be an increasing sequence of finite subsets of $I$ such that $I_{k} \uparrow I$. For each $k$ let $\Lambda_{k}$ be the subset of $\omega$ in $\Lambda$ such that either $\omega(0)=\partial$ or $\omega(0)$ is in $I_{k}$. The dead trajectory $\delta_{k}$ is the unique $\omega$ in $\Lambda_{k}$ such that $\omega(0)=\partial$. Define $J_{k}: \Lambda_{k+1} \rightarrow \Lambda_{k}$ by

$$
J_{k} \omega(n)=\left\{\begin{array}{ccc}
\partial & \text { if } & \sigma\left(I_{k}\right)=+\infty \\
\omega\left(\sigma\left(I_{k}\right)+n\right) & \text { if } & \sigma\left(I_{k}\right)<+\infty .
\end{array}\right.
$$

The inverse limit $\Lambda_{\infty}^{0}$ is the collection of sequences $\left\{\omega_{k}\right\}_{1 \leqq k \leqq \infty}$ with each $\omega_{k}$ in $\Lambda_{k}$ and such that $J_{k} \omega_{k+1}=\omega_{k}$ for all $k$. The extended sample space is reduced inverse limit $\Lambda_{\infty}=\Lambda_{\infty}^{0}-\{\delta\}$ where $\delta$ is the point in $\Lambda_{\infty}^{0}$ whose components are the dead trajectories $\delta_{k}$. We denote by $J_{\infty, k}$ the natural projection of $\Lambda_{\infty}$ onto $\Lambda_{k}$. 
Now consider $h, h^{\prime}>0$ excessive and for each $k$ define the measure $\mathfrak{B}^{(k)}$ on $\Lambda_{k}$ by

$$
\mathscr{E}^{(k)} \xi=\sum \alpha(x) h^{\prime}(x) L_{k}^{h^{\prime}}(x) h(x) \xi_{x}^{h} \xi .
$$

(Here and below we replace a subscript or superscript $I_{k}$ by $k$ for typographical convenience.) Clearly $\mathfrak{B}_{x}{ }^{h}\left[\sigma^{*}\left(I_{k}\right)<+\infty\right]=1$ and so (1.9) yields for $\varphi \geqq 0$ on $\boldsymbol{I}$

$$
\begin{aligned}
& \mathbb{6}^{(k+1)}\left\{\sigma\left(I_{k}\right)<+\infty ; \varphi\left(X_{\sigma\left(I_{k}\right)}\right)\right\}=\sum \alpha(y) h(y) L_{k}^{h}(y) h^{\prime}(y) \oiint_{y}^{h^{\prime}} \varphi\left(X_{\sigma^{*}\left(I_{k}\right)}\right) \\
& =\xi^{(k)} \varphi\left(X_{0}\right)
\end{aligned}
$$

and from this it follows easily that

$$
\xi^{(k+1)} \xi \circ J_{k}=\xi^{(k)} \xi
$$

for $\xi \geqq 0$ on $\Lambda_{k}$ and vanishing on $\delta_{k}$. Thus by a trivial modification of the argument on page 138 of [15],

Theorem 1.3. Let $h, h^{\prime}>0$ be excessive. Then there exists a unique measure $\mathfrak{B}^{h}{ }^{\prime}$, on the extended sample space $\Lambda_{\infty}$ such that

$$
\oiint_{h^{\prime}}^{h}\left[\xi \circ J_{\infty, k}\right]=\sum_{x} \alpha(x) h^{\prime}(x) L_{k}^{h^{\prime}}(x) h(x) \oiint_{x}^{h} \xi
$$

for $\xi \geqq 0$ defined on $\Lambda_{k}$ and vanishing on $\delta_{k}$.

Let $\Phi$ be the natural mapping of the standard sample space $\Lambda \cap\left[X_{0} \neq \partial\right]$ into the extended sample space $\Lambda_{\infty}$ determined by the relations

$$
\begin{array}{rll}
J_{\infty, k} \Phi \omega=\delta_{k} & \text { if } & \sigma\left(I_{k}\right)=+\infty \\
J_{\infty, k} \Phi \omega(n)=\omega\left(\sigma\left(I_{k}\right)+n\right) & \text { if } & \sigma\left(I_{k}\right)<+\infty .
\end{array}
$$

If $h^{\prime}=N(\cdot, x) / \alpha(x)$, then $\alpha h^{\prime} L^{h^{\prime}}{ }_{I_{k}}$ is $\epsilon_{x}$, the point mass located at $x$ when $I_{k}$ contains $x$ and so

$$
h(x) \xi_{x}^{h} \xi \circ \Phi=\mathfrak{E}_{N(\cdot, x) / \alpha(x)}^{h} \xi
$$

for $\xi \geqq 0$ on $\Lambda_{\infty}$.

For future convenience we introduce trajectory variables for sample paths $\omega$ in $\Lambda_{\infty}$ which are parametrized by an artificial two sided time scale. Let $k_{0}(\omega)$ be the smallest integer $k$ such that $J_{\infty, k} \omega \neq \delta_{k}$. For $n \geqq 0$ define

$$
X_{n}(\omega)=J_{\infty, k_{0}} \omega(n) \text {. }
$$

For $n<0$ there exists at most one integer $k_{n}>k_{0}$ such that

Define

$$
\begin{aligned}
\sigma\left(I_{k_{0}}, J_{\infty, k_{n}} \omega\right) & \geqq|n| \\
\sigma\left(I_{k_{0}}, J_{\infty, k_{n}-1} \omega\right) & <|n| .
\end{aligned}
$$

$$
X_{n}(\omega)=\left\{\begin{array}{cl}
J_{\infty, k_{n}} \omega\left(\sigma\left(I_{k_{0}}\right)+n\right) & \text { if } k_{n} \text { exists } \\
\partial & \text { otherwise. }
\end{array}\right.
$$


The random times $\zeta, \zeta^{*}, \sigma(E), \sigma^{*}(E)$ are defined on $\omega$ in $\Lambda_{\infty}$ by

$$
\begin{aligned}
\zeta & =\inf \left\{n>0: X_{n}=\partial\right\}, \\
\zeta^{*} & =\sup \left\{n<0: X_{n}=\partial\right\}, \\
\sigma(E) & =\inf \left\{n: X_{n} \text { is in } E\right\}, \\
\sigma^{*}(E) & =\sup \left\{n: X_{n} \text { is in } E\right\},
\end{aligned}
$$

with the understanding that $\zeta, \sigma(E)=+\infty$ and $\zeta^{*}, \sigma^{*}(E)=-\infty$ when not otherwise defined. Before continuing we note the easily verified equation

$$
\mathfrak{E}_{h^{\prime}}^{h} \sum_{n=\zeta^{*}}^{\zeta} \varphi\left(X_{n}\right)=\sum \alpha(x) h(x) h^{\prime}(x) \varphi(x)
$$

valid for $\varphi \geqq 0$ on $I$ which will play an important role in later sections.

The time reversal operator $\rho$ is determined on $\omega$ in $\Lambda_{\infty}$ by the relationship

$$
X_{n}(\rho \omega)=X_{\sigma^{*}\left(I_{k 0}\right)-n}(\omega)
$$

and routine arguments extend Theorem 1.3 to

Theorem 1.4. Let $h, h^{\prime}>0$ be excessive and let $\xi \geqq 0$ on $\Lambda_{\infty}$. Then

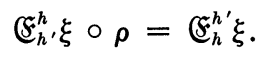

If $h^{\prime}=N(\nu / \alpha)$ with $\nu$ a measure on $I$, then $\sum_{x} \nu(x) h(x) \mathfrak{P}^{h}{ }_{x}$ corresponds to $\mathfrak{B}^{h}{ }_{h^{\prime}}$ via (1.10) and so a special case of Theorem 1.4 can be formulated as

Corollary 1.5. Let $\nu$ be a measure on $I$ such that $N(\nu / \alpha)$ is finite and therefore excessive. Then for any nontrivial excessive $h$ and for $\xi \geqq 0$ on $\Lambda_{\infty}$,

$$
\sum \nu(x) h(x) \mho_{x}^{h} \xi \circ \rho \circ \Phi=\xi_{h}^{N(\nu / \alpha)} \xi .
$$

Now we are ready to establish the Martin representation. Our treatment is only a slight variation of the one given by Hunt in [11]. We first fix a reference point 0 in $I_{1}$ and define the Martin kernel $K(x, y)$ for $x, y$ in $I$ by

$$
K(x, y)=N(x, y) / N(0, y)
$$

Elementary arguments yield the important estimate

$$
\mathfrak{B}_{x}[\sigma(\{0\})<+\infty] \leqq K(x, y) \leqq\left(\mathfrak{P}_{0}[\sigma(\{x\})<+\infty]\right)^{-1} .
$$

We define a metric on $I$ by

$$
d\left(y, y^{\prime}\right)=\sum_{x} \beta(x) \mathfrak{B}_{0}[\sigma\{x\}<+\infty]\left|K(x, y)-K\left(x, y^{\prime}\right)\right|
$$

with $\beta(x)>0$ chosen so that $\sum_{x} \beta(x)<+\infty$. The Martin closure $I^{*}$ is the completion of $\boldsymbol{I}$ with respect to $d$. The Martin boundary is the difference $\Delta=I^{*}-I$. The Martin kernel $K(x, y)$ extends by continuity in an obvious way to $I \times I^{*}$. 
Theorem 1.6. Let $h>0$ be harmonic with $h(0)=1$.

(i) The distribution of $X_{\sigma^{*}\left(I_{k}\right)}$ relative to $\mathfrak{P}^{h}{ }_{0}$ is $N(0, \cdot) L_{{ }_{k}}^{h} h$.

(ii) $X_{\infty}=\operatorname{Lim} X_{n}(n \uparrow \infty)$ exists [a.e. $\mathfrak{P}^{h}{ }_{0}$ ] as a point in the Martin closure $\boldsymbol{I}^{*}$. Thus the probabilities $N(0, \cdot) L_{I_{k}}^{h}$ h converge vaguely to a probability $\ell^{h}$, the distribution of $X_{\infty}$ relative to $\mathfrak{B}^{h}{ }_{0}$.

(iii) $\ell^{h}$ is concentrated on the Martin boundary $\Delta$ and for $x$ in $I$

$$
h(x)=\int K(x, y) \ell^{h}(d y)
$$

Proof. (i) is a special case of Theorem 1.1-(iv). By Corollary 1.5 the random variables $\left\{X_{\sigma^{*}\left(I_{k}\right)}\right\}_{1 \leqq k<\infty}$ relative to $\mathfrak{B}^{h}{ }_{0}$ have the same distribution as the random variables $\left\{X_{\sigma\left(I_{k}\right)}\right\}$ relative to $\underbrace{N(\cdot, 0) / \alpha(0)}_{h}$. For fixed $x$

$$
\begin{aligned}
K(x, y) & =N(x, y) / N(0, y) \\
& =N(y, x) \alpha(0) / N(y, 0) \alpha(x)
\end{aligned}
$$

is $N(\cdot, 0)$ excessive as $y$ varies and so $\left\{K\left(x, X_{\sigma^{*}\left(I_{k}\right)}\right)\right\}_{1 \leqq k<\infty}$ is a reversed supermartingale relative to $\mathfrak{B}_{0}^{h}$. By (1.14) this reversed supermartingale is uniformly bounded and so

$$
\operatorname{Lim} K\left(x, X_{\sigma^{*}\left(I_{k}\right)}\right) \quad(k \uparrow \infty)
$$

exists [a.e. $\mathfrak{P}^{h}{ }_{0}$ ]. It follows from the definition of the Martin closure that $X_{\infty}=$ $\operatorname{Lim} X_{\sigma^{*}\left(I_{k}\right)}(k \uparrow \infty)$ exists $\left[\right.$ a.e. $\mathfrak{P}^{h}{ }_{0}$ ] as a point in the Martin closure $I^{*}$. An application of the well known crossings inequality [12, Chap. 3] shows that actually $X_{n} \rightarrow X_{\infty}$ as $n \uparrow \infty\left[\right.$ a.e. $\left.\mathfrak{B}^{h}{ }_{0}\right]$ and (ii) is proved. That $\ell^{h}$ is concentrated on the Martin boundary $\Delta$ follows from the transience of $\mathfrak{B}^{h}{ }_{0}$. Finally for $x$ in $\boldsymbol{I}$

$$
\begin{aligned}
\xi_{0}^{h} K\left(x, X_{\sigma^{*}\left(I_{k}\right)}\right) & =\sum_{y} N(0, y) L_{k}^{h}(y) h(y) K(x, y) \\
& =\sum_{y} N(x, y) L_{k}^{h}(y) h(y) \\
& =H^{k} h(x)
\end{aligned}
$$

by Theorem 1.1-(ii). As $k \uparrow \infty$ the last expression increases to $h(x)$ and the first expression converges to $\mathbb{E}^{h} K\left(x, X_{\infty}\right)=\int \ell^{h}(d y) K(x, y)$ by (1.14) and the continuity of $K(x, \cdot)$. This establishes the representation (1.16) and the theorem is proved.

For typographical convenience we write $\ell^{z}(d y)$ instead of $\ell^{K(\cdot, z)}(d y)$ when $K(\cdot, z)$ is harmonic. Also we write $L^{z}$ and $L^{z}{ }_{E}$ instead of $L^{K(\cdot, z)}$ and $L^{K(\cdot, z)}$ for general $z$ in $I^{*}$. We also introduce the special notations

$$
\begin{aligned}
& \ell_{k}^{h}(y)=N(0, y) L_{k}^{h}(y) h(y) \\
& \ell_{k}^{z}(y)=N(0, y) L_{k}^{z}(y) K(y, z) .
\end{aligned}
$$

It is easy to check that $K(x, \cdot) \ell^{h}{ }_{k}$ is the distribution of $X_{\sigma^{*}\left(I_{k}\right)}$ relative to $h(x) \mathfrak{P}^{h}{ }_{x}$ for $h$ excessive, that $X_{\infty}=\operatorname{Lim} X_{n}(n \uparrow \infty)$ exists [a.e. $\mathfrak{P}_{x}^{\boldsymbol{h}}{ }_{x}$ ] for $h$ harmonic and that the distribution of $X_{\infty}$ relative to $h(x) \mathfrak{P}^{h}{ }_{x}$ is $K(x, \cdot) \ell^{h}$ for $h$ harmonic. 
We denote by $\Delta_{0}$ the subset of $z$ in $\Delta$ such that $K(\cdot, z)$ is an extremal harmonic function. That is, $z$ belongs to $\Delta_{0}$ if $K(\cdot, z)$ is harmonic and has no nontrivial decomposition

$$
K(\cdot, z)=h+h^{\prime}
$$

with $h, h^{\prime}$ positive and harmonic. If $h$ harmonic has a representation $h(x)=$ $\int K(x, y) \nu(d y)$ with $\nu$ concentrated on $\Delta$, then

$$
\xi_{x}^{h} \xi=\int \nu(d y) \oiint_{x}^{K(\cdot, y)} \xi
$$

for $\xi \geqq 0$ on $\Lambda$. This easy result is an important tool for our proof of

Theorem 1.7. (i) $z$ in $\Delta$ belongs to $\Delta_{0}$ if and only if $K(\cdot, z)$ is harmonic and $\ell^{z}=\epsilon_{z}$, the point mass concentrated at $z$.

(ii) If $h$ is harmonic, then $\ell^{h}$ is the unique measure concentrated on $\Delta_{0}$ such that the representation (1.16) is valid.

Proof. It follows from (1.18) with $\nu$ replaced by $\ell^{h}$ that

$$
\ell^{h}(d y)=\int \ell^{h}(d w) \ell^{w}(d y)
$$

Consider $z$ in $\Delta$ such that $K(\cdot, z)$ is harmonic. If $K(\cdot, z)$ admits a nontrivial decomposition (1.17) then clearly $\ell^{z}=\ell^{h}+\ell^{h^{\prime}}$ and it follows that if $\ell^{z}=\epsilon_{z}$, then $z$ belongs to $\Delta_{0}$. It follows then from (1.19) that every probability $\ell^{h}$ is concentrated on $\Delta_{0}$. In particular this is true for $\ell^{z}$. Thus if $\ell^{z} \neq \epsilon_{z}$, then $\ell^{z}$ admits a nontrivial decomposition which determines via the representation (1.16) a nontrivial decomposition (1.17) and so $z$ does not belong to $\Delta_{0}$. This proves everything but uniqueness. But if (1.16) is valid with $\ell^{h}$ replaced by a measure $\nu$ concentrated on $\Delta_{0}$, then also (1.19) is valid with $\ell^{h}$ replaced by $\nu$ and it follows directly that actually $\nu=\ell^{h}$.

These results plus the Martingale convergence theorem lead directly to

Theorem 1.8. (Fatou). Let $h>0$ be harmonic and let $x$ be in I. Then as $n \uparrow \infty$

$$
\left.h\left(X_{n}\right) \rightarrow\left(d \ell^{h} / d \ell^{1}\right)\left(X_{\infty}\right) \quad \text { [a.e. } \mathfrak{P}_{x}\right] .
$$

For $x$ in $I$ and $y$ in $I^{*}$ the Naïm kernel $\Theta(x, y)$ is defined by

$$
\Theta(x, y)=K(x, y) / N(x, 0) \text {. }
$$

Clearly

$$
\Theta(x, y)=\Theta(y, x)
$$

for $x, y$ in $I$. In order to extend the definition of $\Theta(x, y)$ to $x$ in $I^{*}$ we prove

Lemma. 1.9. Let $x, y$ be in $I^{*}$. Then

$$
\sum_{z} \ell_{k}^{x}(z) \Theta(z, y)
$$


increases with $k$. If $y$ belongs to I, then actually

$$
\sum_{z} \ell_{k}^{x}(z) \Theta(z, y) \uparrow \Theta(y, x)
$$

as $k \uparrow \infty$.

Proof. Consider first $y$ in $I$. Then

$$
\begin{aligned}
\sum_{z} \ell_{k}^{x}(z) \Theta(z, y) & =\sum_{z} \ell_{k}^{x}(z) \Theta(y, z) \\
& =\sum_{z} \ell_{k}^{x}(z) K(y, z) / N(y, 0) \\
& =E_{0}^{K(\cdot, x)} K\left(y, X\left(\sigma_{k}^{*}\right)\right) / N(y, 0)
\end{aligned}
$$

which increases to

$$
K(y, x) / N(y, 0)=\Theta(y, x)
$$

as in the proof of Theorem 1.6 and (1.21) is established. To handle general $y$ in $I^{*}$ it suffices to observe that for each $k$

$$
\sum_{z} \sum_{w} \ell_{k}^{x}(z) \ell_{j}^{y}(w) \Theta(w, z) \uparrow \sum_{z} \ell_{k}^{x}(z) \Theta(z, y)
$$

as $j \uparrow \infty$.

Now for $x, y$ in $I^{*}$ define

$$
\Theta(x, y)=\operatorname{Lim} \sum_{z} \ell_{k}^{x}(z) \Theta(z, y) \quad(k \uparrow \infty) .
$$

Obviously $\Theta$ is lower semicontinuous in each variable separately and it follows easily from (1.22) that the symmetry condition (1.20) is valid for $x, y$ in $I^{*}$.

We finish by establishing

$$
\left(\xi_{1}{ }^{1} \varphi\left(X_{-\infty}, X_{+\infty}\right)=\alpha(0) \int \ell^{1}(d y) \int \ell^{1}(d z) \Theta(y, z) \varphi(y, z)\right.
$$

for $\varphi \geqq 0$ on $\Delta_{0} \times \Delta_{0}$. Note first that for $\varphi \geqq 0$ on $\Delta_{0}$ and for $y$ in $\Delta_{0}$

$$
\begin{aligned}
\left(_{\dot{E}^{1}(\cdot, y)}^{1} \varphi\left(X_{+\infty}\right)\right. & =\operatorname{Lim} \sum_{x} \alpha(x) L_{k}^{y}(x) K(x, y) \int \ell^{1}(d z) K(x, z) \varphi(z) \\
& =\operatorname{Lim} \sum_{x} \alpha(x) L_{k}^{y}(x) K(x, y) N(x, 0) \int \ell^{1}(d z) \Theta(x, z) \varphi(z) \\
& =\operatorname{Lim} \alpha(0) \sum_{x} N(0, x) L_{k}^{y}(x) K(x, y) \int \ell^{1}(d z) \Theta(x, z) \varphi(z) \\
& =\operatorname{Lim} \alpha(0) \sum_{x} l_{k}^{y}(x) \int \ell^{1}(d z) \Theta(x, z) \varphi(z) \\
& =\alpha(0) \int \ell^{1}(d z) \Theta(y, z) \varphi(z)
\end{aligned}
$$


with all limits taken as $k \uparrow \infty$. Then by (1.18) the left side of (1.24)

$$
\begin{aligned}
& =\int \ell^{1}(d z) \xi_{1}^{K(\cdot, z)} \varphi\left(X_{-\infty}, z\right) \\
& =\int \ell^{1}(d z) \xi_{K(\cdot, z)}^{1} \varphi\left(z, X_{+\infty}\right)
\end{aligned}
$$

which equals the right side of (1.24) by (1.25).

2. The universal Dirichlet space. The universal Dirichlet form $\mathcal{E}(f, f)$ is defined by

$$
\mathcal{E}(f, f)=\frac{1}{2} \sum_{x, y} \alpha(x) P(x, y)\{f(x)-f(y)\}^{2}
$$

when it converges. The universal Dirichlet space $\mathfrak{F}$ is the set of functions $f$ on $I$ for which (2.1) converges. By the symmetry condition (1.2)

$$
\sum_{x, y} \alpha(x) P(x, y) f(x) f(y) \leqq \sum_{x} \alpha(x) f^{2}(x)
$$

and it follows that $P$ is a contraction on $L^{2}(\alpha)$. Thus

Theorem 2.1. $L^{2}(\alpha)$ belongs to $\mathcal{F}$ and

$$
\mathcal{E}(f, g)=\sum_{x} \alpha(x) f(x)\{g-P g\}(x)
$$

for $f, g$ in $L^{2}(\alpha)$.

If $\varphi \geqq 0$ has finite support, then $N \varphi$ is bounded and therefore

$$
\sum_{x} \alpha(x) \varphi(x) N \varphi(x)<+\infty \text {. }
$$

But then

$$
\sum_{x} \alpha(x) \varphi(x) P^{2 n} \varphi(x)=\sum_{x} \alpha(x)\left\{P^{n} \varphi(x)\right\}^{2} \rightarrow 0
$$

as $n \uparrow \infty$ and therefore

Lemma 2.2. $\quad P^{n} \rightarrow 0$ in the strong operator topology on $L^{2}(\alpha)$.

A somewhat deeper result is

Theorem 2.3. Let $\varphi \geqq 0$ satisfy (2.3).

(i) $f=N \varphi$ belongs to $\mathcal{F}$ and

$$
\begin{aligned}
\mathcal{E}(f, f) & =\sum_{x} \alpha(x) \varphi(x) f(x) \\
& =\sum_{x, y} \alpha(x) \varphi(x) N(x, y) \varphi(y)
\end{aligned}
$$

(ii) $f_{n}=\sum_{0 \leqq k \leqq n} P^{k} \varphi$ belongs to $L^{2}(\alpha)$ and $\varepsilon\left(f-f_{n}, f-f_{n}\right) \rightarrow 0$ as $n \uparrow \infty$. 
Proof. Obviously (i) will follow from (ii) and (ii) will follow if we show that $\mathcal{E}\left(f_{m}-f_{n}, f_{m}-f_{n}\right) \rightarrow 0$ as $m, n \uparrow \infty$. But for $m<n$

$$
\begin{aligned}
\mathcal{E}\left(f_{n}\right. & \left.-f_{m}, f_{n}-f_{m}\right) \\
& =\sum_{x} \alpha(x)\left\{f_{n}(x)-f_{m}(x)\right\}(1-P)\left\{f_{n}-f_{m}\right\}(x) \\
& =\sum_{x} \alpha(x)\left\{f_{n}(x)-f_{m}(x)\right\}\left\{P^{m+1} \varphi(x)-P^{n+1} \varphi(x)\right\} \\
& =\sum_{x} \alpha(x) \varphi(x)\left\{\sum_{k=m+1}^{m+1+n} P^{k} \varphi(x)-\sum_{k=m+1}^{m+1+m} P^{k} \varphi(x)-\sum_{k=n+1}^{n+1+n} P^{k} \varphi(x)+\sum_{k=n+1}^{n+1+m} P^{k} \varphi(x)\right\} \\
& \leqq \sum_{x} \alpha(x) \varphi(x) \sum_{k=m+1}^{\infty} P^{k} \varphi(x)
\end{aligned}
$$

which $\downarrow 0$ as $m \uparrow \infty$ because of (2.3).

Corollary 2.4. Let $\varphi \geqq 0$ satisfy (2.3) and set $g=N \varphi$. Then

$$
\mathcal{E}(f, g)=\sum_{x} \alpha(x) \varphi(x) f(x)
$$

for $f$ in $L^{2}(\alpha)$ or for $f=N \psi$ with $\psi \geqq 0$ satisfying (2.3).

Proof. With $g_{n}$ as in Theorem 2.3 and for $f$ in $L^{2}(\alpha)$

$$
\begin{aligned}
\mathcal{E}\left(g_{n}, f\right) & =\sum_{x} \alpha(x) f(x)\left\{g_{n}(x)-P g_{n}(x)\right\} \\
& =\sum_{x} \alpha(x) f(x)\left\{\varphi(x)-P^{n+1} \varphi(x)\right\}
\end{aligned}
$$

and (2.4) follows upon letting $n \uparrow \infty$ because of Theorem 2.3-(ii) and Lemma 2.2. The extension to $f=N \psi$ follows after another passage to the limit, using Theorem 2.3 (ii).

Remark. (2.4) is not true for general $f$ in $\mathcal{F}$. In particular it fails for $f=1$.

The capacity of a set $E$ is defined for $E$ finite by

$$
\operatorname{Cap}(E)=\sum_{x} \alpha(x) L_{E}^{1}(x) .
$$

For general $E$ the capacity is defined by

$$
\operatorname{Cap}(E)=\sup \{\operatorname{Cap}(F): F \subset E, F \text { finite }\} \text {. }
$$

An important interpretation for capacity is established in

Theorem 2.5. If $H^{E} 1$ is a potential, then

$$
\operatorname{Cap}(E)=\mathfrak{P}_{1}^{1}[\sigma(E)<+\infty]=\sum \alpha(x) L_{E}^{1}(x)
$$

Proof. If $H^{E} 1$ is a potential, then by Theorem 1.1 


$$
\begin{aligned}
\sum_{x} \alpha(x) L_{k}^{1}(x) P_{x}[\sigma(E)<+\infty] & =\sum_{x} \alpha(x) L_{k}^{1}(x) N L_{E}^{1}(x) \\
& =\sum_{y} \alpha(y) L_{E}^{1}(y) N L_{k}^{1}(y) \\
& =\sum_{y} \alpha(y) L_{E}^{1}(y) H^{k} 1(y)
\end{aligned}
$$

and the theorem follows upon letting $k \uparrow \infty$ and approximating $E$ by finite subsets.

The basic connection between capacity and the Dirichlet form $\varepsilon$ is established in

Theorem 2.6. If $f$ is in $L^{2}(\alpha)$ and if $|f| \geqq 1$ on $E$, then

$$
\varepsilon(f, f) \geqq \operatorname{Cap}(E) \text {. }
$$

Proof. Clearly $\mathcal{E}(f, f) \geqq \varepsilon(|f|,|f|)$ and so we can assume $f \geqq 0$. Also it suffices to consider $E$ finite. But then Corollary 2.4 is applicable with $\varphi=L^{1}{ }_{E}$ and so

$$
\begin{aligned}
\mathcal{E}(f, f)= & \mathcal{E}\left(H^{E} 1, H^{E} 1\right)+\mathcal{E}\left(f-H^{E} 1, f-H^{E} 1\right) \\
& +2 \mathcal{E}\left(H^{E} 1, f-H^{E} 1\right) \\
& \geqq \sum_{x} \alpha(x) L_{E}(x)\left\{H^{E} 1(x)+2\left(f-H^{E} 1\right)\right\}(x) \\
& \geqq \operatorname{Cap}(E) .
\end{aligned}
$$

Remark. Again (2.5) fails for general $f$ in $F$, with $f=1$ being the obvious counterexample.

Corollary 2.7. Let $f$ be in $L^{2}(\alpha)$ and let $\epsilon>0$. Then

$$
\text { Cap }\{x:|f(x)| \geqq \epsilon\} \leqq\left(1 / \epsilon^{2}\right) \varepsilon(f, f) .
$$

Proof. Apply (2.5) with $f$ replaced by $(1 / \epsilon) f$.

Corollary 2.8. For $x$ in $I$ and $f$ in $L^{2}(\alpha)$

$$
\alpha(x) \mathfrak{B}_{x}\left[\sigma_{(x)}^{+}=+\infty\right]\{f(x)\}^{2} \leqq \varepsilon(f, f) .
$$

Proof. Apply Theorem 2.6 with $E=\{x\}$.

Corollary 2.9. Let $\left\{f_{n}\right\}$ be a sequence in $L^{2}(\alpha)$ such that

$$
\varepsilon\left(f_{m}-f_{n}, f_{m}-f_{n}\right) \rightarrow 0
$$

as $m, n \uparrow \infty$. Then there exists a finite function $f$ such that

(i) $f_{n} \rightarrow f$ pointwise.

(ii) $\varepsilon(f, f)<+\infty$ and $\varepsilon\left(f-f_{n}, f-f_{n}\right) \rightarrow 0$.

Proof. (i) follows from Corollary 2.8 and (ii) follows from (i) and Fatou's lemma.

Now we are ready for 
2.1. Definition. $f$ in $\mathcal{F}$ belongs to the minimal Dirichlet space $\mathfrak{F}^{\mathrm{min}}$ if there exist $f_{n}, n \geqq 1$ in $L^{2}(\alpha)$ such that

2.1.1. $f_{n} \rightarrow f$ pointwise

2.1.2. $\varepsilon\left(f-f_{n}, f-f_{n}\right) \rightarrow 0$.

Corollary 2.9 leads directly to

Theorem 2.10. (i) The minimal Dirichlet space $\mathfrak{F}^{\mathrm{min}}$ is a Hilbert space relative to the inner product $\varepsilon(f, g)$.

(ii) The relations (2.4), (2.5), (2.6) and (2.7) are valid for $f$ in $\mathcal{F}^{\mathrm{min}}$.

Remark. The situation is quite different if the $\operatorname{road} \operatorname{map} P$ is recurrent. The function 1 can be approximated relative to $\varepsilon$ by functions in $L^{2}(\alpha)$ and so no Hilbert space completion of $L^{2}(\alpha)$ relative to $\varepsilon$ exists. For an indirect proof see the remark following Theorem 5.1.

Notice that the full Dirichlet space $\mathfrak{F}$ cannot possibly be a Hilbert space since 1 belongs to $\mathcal{F}$ and $\varepsilon(1,1)=0$.

The basic decomposition of $\mathfrak{F}$ is established in

Theorem 2.11. (i) $\mathcal{E}(f, h)=0$ for $f$ in $\mathfrak{F}^{\mathrm{min}}$ and $h$ in $\mathfrak{F}$ harmonic.

(ii) If $g$ belongs to $\mathfrak{F}$, then $H^{I-I_{k}} g$ is well defined and belongs to $\mathfrak{F}$ for all $k$,

$$
H g=\operatorname{Lim} H^{I-I_{k}} g \quad(k \uparrow \infty)
$$

exists pointwise and $\mathrm{Hg}$ is the unique harmonic function in $\mathcal{F}$ such that $\mathrm{g}-\mathrm{Hg}$ belongs to $\mathfrak{F}^{\mathrm{min}}$. Also $g-H^{I-I_{k}} g$ belongs to $\mathfrak{F}^{\mathrm{min}}$ for all $k$ and

$$
g-H^{I-I_{k}} g \rightarrow g-H g
$$

relative to the inner product $\mathcal{E}$ as $k \uparrow \infty$.

Proof. First consider $f$ in $\mathcal{F}^{\text {min }}$ supported by $D$ a finite subset of $I$, let $M=$ $I-D$ and let $h$ be a bounded function in $\mathcal{F}$ such that $H^{M} h=h$. Then

$$
\begin{aligned}
\varepsilon(f, h)= & \frac{1}{2} \sum_{x \text { in } D} \sum_{y \text { in } D} \alpha(x) P(x, y)\{f(x)-f(y)\}\{h(x)-h(y)\} \\
& +\sum_{x \text { in } D} \sum_{y \text { in } M} \alpha(x) P(x, y) f(x)\{h(x)-h(y)\} \\
= & \sum_{x \text { in } D} \alpha(x)\left\{f(x) h(x) P 1_{D}(x)-f(x) P 1_{D} h(x)\right\} \\
& +\sum_{x \text { in } D} \alpha(x)\left\{f(x) h(x) P 1_{M}(x)-f(x) P 1_{M} h(x)\right\} \\
= & 0
\end{aligned}
$$

since $P h=h$ on $D$ by the "optional stopping time theorem" for martingales [12, Chap. 3]. It follows that for bounded $g$ in $\mathcal{F}$

$$
\begin{gathered}
\varepsilon(g, g)= \\
\varepsilon\left(g-H^{I-I_{k}} g, g-H^{I-I_{k}} g\right) \\
+\varepsilon\left(H^{I-I_{k}} g, H^{I-I_{k}} g\right)
\end{gathered}
$$


and the theorem follows easily with the help of truncation and passage to the limit.

Finally we supplement Theorem 2.5 with

Theorem 2.12. If Cap $(E)<+\infty$ then $H^{E} 1$ is a potential, $H^{E} 1$ belongs to $\mathfrak{F}^{\mathrm{min}}$ and

$$
\operatorname{Cap}(E)=\varepsilon\left(H^{E} 1, H^{E} 1\right) .
$$

Proof. That $H^{E} 1$ belongs to $\mathfrak{F}^{\mathrm{min}}$ and that (2.9) is valid follow from Theorem 2.3. That $H^{E} 1$ is a potential then follows from Theorem 2.11-(i).

3. Formulae for Dirichlet norms. Fix a finite set $D$, let $M=\boldsymbol{I}-D$ and consider the "killed" operators

$$
\begin{aligned}
& P^{D}(x, y)=1_{D}(x) P(x, y) 1_{D}(y) \\
& N^{D}(x, y)=1_{D}(x) \sum_{k=0}^{\infty}\left(P^{D}\right)^{k}(x, y) 1_{D}(y)
\end{aligned}
$$

and the corresponding ones with $D$ replaced by $M$.

Clearly

$$
\begin{gathered}
\alpha(x) N^{D}(x, y)=\alpha(y) N^{D}(y, x), \\
N(x, y)=N^{D}(x, y)+H^{M} N(x, y),
\end{gathered}
$$

and therefore

$$
\alpha(x)\left(H^{M} N\right)(x, y)=\alpha(y)\left(H^{M} N\right)(y, x) .
$$

Some elementary identities are collected in

Lemma 3.1. (i) For $x, w$ in $D$ and $y$ in $M$,

$$
\begin{gathered}
\mathfrak{P}_{x}\left[\sigma(M)=n+1 ; \quad X_{\sigma(M)-1}=w ; \quad X_{\sigma(M)}=y\right]=\left(P^{D}\right)^{n}(x, w) P(w, y), \\
\mathfrak{P}_{x}\left[X_{\sigma(M)-1}=w ; \quad X_{\sigma(M)}=y\right]=N^{D}(x, w) P(w, y), \\
H^{M}(x, y)=N^{D} P(x, y) .
\end{gathered}
$$

(ii) For $x$ in $D$

$$
P 1_{M}(x)=\sum_{n=0}^{\infty}\left\{P N^{M} P N^{D}\right\}^{n} L_{D}^{1}(x) .
$$

(iii) For $x, w$ in $D$

$$
N(x, y)=\sum_{n=0}^{\infty} N^{D}\left\{P N^{M} P N^{D}\right\}^{n}(x, w) .
$$

(iv) For $x$ in $D$ and $y$ in $M$

$$
N(x, y)=\sum_{n=0}^{\infty} N^{D}\left\{P N^{M} P N^{D}\right\}^{n} P N^{M}(x, y) .
$$


(v) For $y, z$ in $M$

$$
N(y, z)=\sum_{n=0}^{\infty} N^{M}\left\{P N^{D} P N^{M}\right\}(y, z) .
$$

(vi) For $x$ in $D$

$$
N L_{D}^{1}(x)=1 ; \quad N^{D} P 1_{M}(x)=1 .
$$

These identities have obvious probabilistic interpretations which in turn suggest proofs. We omit the details.

Entrance and return times for excursions into $D$ are defined by

$$
\begin{aligned}
& e(1)=\sigma(D) \\
& r(1)=\inf \left\{n>e(1): X_{n} \text { is in } M\right\} \\
& e(2)=\inf \left\{n>r(1): X_{n} \text { is in } D\right\}
\end{aligned}
$$

etc.

with the understanding that $e(i), r(i)=+\infty$ when not otherwise defined. The local Naïm kernel is defined on $M \times M$ by

$$
\Theta^{M}(y, z) \alpha(y) \alpha(z)=\sum_{x \text { in } D} \alpha(x) H^{M}(x, y) P(x, z)
$$

or equivalently,

$$
\Theta^{M}(y, z) \alpha(y) \alpha(z)=\sum_{x, w \text { in } D} N^{D}(x, w) P(x, y) P(w, z)
$$

from which symmetry of $\Theta^{M}$ is obvious. The universal Dirichlet form on $M$ is denoted and defined by

$$
\frac{1}{2} \Theta^{M}\langle f, f\rangle=\frac{1}{2} \sum_{y, z \text { in } M} \Theta^{M}(y, z) \alpha(y) \alpha(z)\{f(y)-f(z)\}^{2} .
$$

The killed Dirichlet space $\mathfrak{F}^{D}$ is defined by

$$
\mathfrak{F}^{D}=\{f \varepsilon F: f(y)=0 \text { for } y \text { in } M\} .
$$

and the killed Dirichlet form $\mathcal{E}^{D}$ is defined by

$$
\begin{aligned}
\mathcal{E}^{D}(f, f)= & \frac{1}{2} \sum_{x, w \text { in } D} \alpha(x) P(x, w)\{f(x)-f(w)\}^{2} \\
& +\sum_{x \text { in } D} \alpha(x) P 1_{M}(x) f^{2}(x) .
\end{aligned}
$$

Obviously $\mathfrak{F}^{D}$ is contained in the minimal Dirichlet space $\mathfrak{F}^{\mathrm{min}}$ and

$$
\varepsilon^{D}(f, g)=\varepsilon(f, g)
$$

for $f, g$ in $\mathcal{F}^{D}$. The Dirichlet form reflected on $D$ is denoted and defined by

$$
\begin{aligned}
\mathcal{E}^{D, r}(f, f)= & \frac{1}{2} \sum_{x, w \text { in } D} \alpha(x) P(x, w)\{f(x)-f(w)\}^{2} \\
& +\sum_{x \text { in } D} \sum_{y \text { in } M} \alpha(x) P(x, y)\{f(x)-f(y)\}^{2} .
\end{aligned}
$$


Important results involving these forms are collected in

Theorem 3.2. (i) For $f$ defined on $M$

$$
\frac{1}{2}\left(\mathfrak{\xi}_{1}^{1} \sum_{i=0}^{\infty}\left\{f\left(X_{r(i)}\right)-f\left(X_{e(i)-1}\right)\right\}^{2}=\frac{1}{2} \Theta^{M}\langle f, f\rangle .\right.
$$

If these expressions are finite, then $H^{M} f$ converges and also

$$
\frac{1}{2} \xi_{1}^{1} \sum_{i=0}^{\infty}\left\{f\left(X_{r(i)}\right)-f\left(X_{e(i)-1}\right)\right\}^{2}=\varepsilon^{D, r}\left(H^{M} f, H^{M} f\right) .
$$

(ii) $\mathcal{E}^{D, r}(f, f)=\mathcal{E}^{D}(f, f)$ for $f$ in the killed Dirichlet space $\mathfrak{F}^{D}$.

(iii) $\mathcal{F}^{D, r}(f, h)=0$ for $f$ in $\mathcal{F}^{D}$ and for $h$ in $\mathcal{F}$ satisfying $H^{M} h=h$.

(iv) If $f$ belongs to $\mathcal{F}$, then $f-H^{M} f$ belongs to $\mathfrak{F}^{D}$ and

$$
\varepsilon^{D, r}(f, f)=\varepsilon^{D}\left(f-H^{M} f, f-H^{M} f\right)+\frac{1}{2} \Theta^{M}\langle f, f\rangle .
$$

(v) For $f$ defined on $I$

$$
\begin{aligned}
\sum_{x \text { in } D} \sum_{y} \alpha(x) P(x, y)\{f(x)-f(y)\}^{2} & =\xi_{1}^{1} \sum_{i=0}^{\infty}\left\{f\left(X_{r(i)}\right)-f\left(X_{r(i)-1}\right)\right\}^{2} \\
& =\xi_{1}^{1} \sum_{i=0}^{\infty}\left\{f\left(X_{e(i)}\right)-f\left(X_{e(i)-1}\right)\right\}^{2} \\
\sum_{x \text { in } D} \sum_{y \text { in I }} \alpha(x) P(x, y)\{f(x)-f(y)\}^{2} & =\xi_{1}^{1} \sum_{n=-\infty}^{+\infty} 1_{D}\left(X_{n}\right)\left\{f\left(x_{n+1}\right)-f\left(X_{n}\right)\right\}^{2} .
\end{aligned}
$$

(vi) For $f$ defined on $I$

$$
\begin{aligned}
\mathcal{E}^{D, r}(f, f)= & \frac{1}{2} \xi_{1}^{1} \sum_{n=-\infty}^{+\infty} 1_{D}\left(X_{n}\right)\left\{f\left(X_{n+1}\right)-f\left(X_{n}\right)\right\}^{2} \\
& +\frac{1}{2} \xi_{1}^{1} \sum_{i=0}^{\infty}\left\{f\left(X_{e(i)}\right)-f\left(X_{e(i)-1}\right)\right\}^{2}
\end{aligned}
$$

Proof. Consider first

$\xi_{1}^{1} \sum_{i=0}^{\infty}\left\{f\left(X_{r(i)}\right)-f\left(X_{e(i)-1}\right)\right\}^{2}=\sum_{i=0}^{\infty} \xi_{1}^{1} \sum_{y \text { in } M} H^{M}\left(X_{e(i)}, y\right)\left\{f(y)-f\left(X_{e(i)-1}\right)\right\}^{2}$

which after application of the time reversal identity (1.12) can be written

$$
\begin{aligned}
\sum_{i=0}^{\infty} \xi_{1}^{1} \sum_{y \text { in } M} H^{M} & \left(X_{r(i)-1}, y\right)\left\{f(y)-f\left(X_{r(i)}\right)\right\}^{2} \\
& =\sum_{i=0}^{\infty} \xi_{1}^{1} \sum_{x \text { in } D} N^{D}\left(X_{e(i)}, x\right) \sum_{y, z \text { in } M} P(x, z) H^{M}(x, y)\{f(y)-f(z)\}^{2} \\
& =\sum_{x, w \text { in } D} \alpha(w) P 1_{M}(w) N^{D}(w, x) \sum_{y, z \text { in } M} P(x, z) H^{M}(x, y)\{f(y)-f(z)\}^{2} \\
& =\Theta^{M}\langle f, f\rangle
\end{aligned}
$$


proving the first identity in (i). In establishing the second identity it suffices by truncation and passage to the limit to consider $f$ bounded so that $H^{M} f$ is automatically well defined. For typographical convenience we put $g=H^{M} f$ and note first that by the martingale property of $g\left(X_{n}\right)$,

$$
\begin{aligned}
\frac{1}{2} \xi_{1}^{1} \sum_{i=0}^{\infty} & \left\{g\left(X_{r(i)}\right)-g\left(X_{e(i)-1}\right)\right\}^{2} \\
= & \frac{1}{2} \xi_{1}^{1} \sum_{i=0}^{\infty}\left\{g\left(X_{r(i)}\right)-g\left(X_{e(i)}\right)\right\}^{2} \\
& +\frac{1}{2} \xi_{1}^{1} \sum_{i=0}^{\infty}\left\{g\left(X_{e(i)}\right)-g\left(X_{e(i)-1}\right)\right\}^{2} .
\end{aligned}
$$

But

and

$$
\begin{aligned}
\mathfrak{E}_{1}^{1} \sum_{i=0}^{\infty} & \left\{g\left(X_{e(i)}\right)-g\left(X_{e(i)-1}\right)\right\}^{2} \\
& =\mathfrak{F}_{1}^{1} \sum_{i=0}^{\infty}\left\{g\left(X_{r(i)}\right)-g\left(X_{r(i)-1}\right)\right\}^{2} \\
& =\sum_{x, w \text { in } D} \sum_{y \text { in } M} \alpha(w) P 1_{M}(w) N^{D}(w, x) P(x, y)\{g(y)-g(x)\}^{2} \\
& =\sum_{x \text { in } D} \sum_{y} \alpha(x) P(x, y)\{g(x)-g(y)\}^{2}
\end{aligned}
$$

$$
\begin{aligned}
\xi_{1}^{1} \sum_{i=0}^{\infty} & \left\{g\left(X_{r(i)}\right)-g\left(X_{e(i)}\right)\right\}^{2} \\
& =\sum_{x \text { in } D} \sum_{y \text { in } M} \alpha(x) P 1_{M}(x) H^{M}(x, y)\{g(x)-g(y)\}^{2} \\
& =\sum_{x \text { in } D} \alpha(x) P 1_{M}(x)\left\{H^{M} g^{2}(x)-g^{2}(x)\right\} \\
& =\sum_{x \text { in } D} \alpha(x)\left\{P 1_{M}(x) N^{D} P 1_{M} g^{2}(x)-P 1_{M}(x) g^{2}(x)\right\} \\
& =\sum_{x} \alpha(x)\left\{1_{D}(x) P 1_{M} g^{2}(x)-1_{D}(x) P 1_{M}(x) g^{2}(x)\right\} \\
& =\sum_{x} \alpha(x)\left\{1_{D}(x) P g^{2}(x)-1_{D}(x) P 1_{D} g^{2}(x)-1_{D}(x) g^{2}(x) P 1_{M}(x)\right\} \\
& =\sum_{x} \alpha(x)\left\{1_{D}(x) P g^{2}(x)-1_{D}(x) g^{2}(x) P 1_{D}(x)-1_{D}(x) g^{2}(x) P 1_{M}(x)\right\} \\
& =\sum_{x \text { in } D} \alpha(x)\left\{P g^{2}(x)-g^{2}(x)\right\} \\
& =\sum_{x \text { in } D} \sum_{y} \alpha(x) P(x, y)\{g(y)-g(x)\}^{2}
\end{aligned}
$$

and the second part of (i) is proved. (In the last step we used the identity $g=P g$ on $D$ already noted in the proof of Theorem 2.11.) Before continuing we remark that a shorter but in our opinion less illuminating proof can be 
given by working instead with $\Theta^{M}\langle g, g\rangle$ and using the identity

$$
\begin{aligned}
& \sum_{x \text { in } D} \sum_{y, z \text { in } M} \alpha(x) H^{M}(x, y) P(x, z)\{g(y)-g(z)\}^{2} \\
& \quad=\sum_{x \text { in } D} \sum_{y, z \text { in } M} \alpha(x) H^{M}(x, y) P(x, z)\left\{[g(y)-g(x)]^{2}+[g(x)-g(z)]^{2}\right\} .
\end{aligned}
$$

The first identity in (v) is the same as (3.11), the second follows from (1.11) and then (vi) follows upon combining the identities in (v). The identity (ii) is obvious and (iv) will follow from (i) and (ii) once (iii) is established. The easiest way to prove (iii) is to argue as in the proof of Theorem 2.11. However we prefer to give a proof of (ii), (iii) and (iv) together which is based on (vi) and has a more probabilistic flavor. For this purpose consider $f$ in $F$, define $\varphi=\left(1-P^{D}\right)$ $\left(f-H^{M} f\right)$ and note the easily verified identities

$$
\begin{gathered}
f-H^{M} f=N^{D} \varphi \\
\mathcal{E}^{D}\left(f-H^{M} f, f-H^{M} f\right)=\sum_{x \text { in } D} \alpha(x) \varphi(x)\left\{f-H^{M} f\right\}(x) .
\end{gathered}
$$

The process

$$
M^{D} f(n)=f\left(X_{n}\right)+\sum_{k=-\infty}^{n-1} 1_{D}\left(X_{k}\right) \varphi\left(X_{k}\right)
$$

satisfies the identity

$$
\begin{aligned}
& \mathfrak{\xi}_{1}^{1} \sum_{n=-\infty}^{+\infty} 1_{D}\left(X_{n}\right)\{M f(n+1)-M f(n)\}^{2} \\
& =\xi_{1}^{1} \sum_{i=0}^{\infty}\left\{M f\left(X_{r(i)}\right)-M f\left(X_{e(i)}\right)\right\}^{2} .
\end{aligned}
$$

This identity can best be understood in the context of a local martingale property for the process (3.15) along excursions into $D$, but it is most easily checked by direct computation using the identity

$$
\xi_{x}\left\{f\left(X_{\sigma(M)}\right)-f(x)+\sum_{k=0}^{\sigma(M)-1} \varphi\left(X_{k}\right)\right\}^{2}=\bigotimes_{x} \sum_{k=0}^{\sigma(M)-1}\left\{f\left(X_{k+1}\right)-f\left(X_{k}\right)+\varphi\left(X_{k}\right)\right\}^{2}
$$

for $x$ in $D$. The left side of (3.16)

$$
\begin{aligned}
= & \xi_{1}^{1} \sum_{n=-\infty}^{+\infty} 1_{D}\left(X_{n}\right)\left\{f\left(X_{n+1}\right)-f\left(X_{n}\right)\right\}^{2} \\
& +\xi_{1}^{1} \sum_{n=-\infty}^{+\infty} 1_{D}\left(X_{n}\right) \varphi^{2}\left(X_{n}\right) \\
& +2 \xi_{1}^{1} \sum_{n=-\infty}^{+\infty} 1_{D}\left(X_{n}\right) \varphi\left(X_{n}\right)\left\{f\left(X_{n+1}\right)-f\left(X_{n}\right)\right\} \\
= & \xi_{1}^{1} \sum_{n=-\infty}^{+\infty} 1_{D}\left(X_{n}\right)\left\{f\left(X_{n+1}\right)-f\left(X_{n}\right)\right\}^{2} \\
& -\xi_{1}^{1} \sum_{n=-\infty}^{+\infty} 1_{D}\left(X_{n}\right) \varphi^{2}\left(X_{n}\right) .
\end{aligned}
$$


The right side of (3.16)

$$
\begin{aligned}
= & \xi_{1}^{1} \sum_{i=0}^{\infty}\left\{f\left(X_{r(i)}\right)-f\left(X_{e(i)}\right)\right\}^{2} \\
& +\xi_{1}^{1} \sum_{i=0}^{\infty}\left\{\sum_{n=e(i)}^{r(i)-1} \varphi\left(X_{n}\right)\right\}^{2} \\
& +2 \xi_{1}^{1} \sum_{i=0}^{\infty}\left\{f\left(X_{r(i)}\right)-f\left(X_{e(i)}\right)\right\} \sum_{n=e(i)}^{r(i)-1} \varphi\left(X_{n}\right) \\
= & \xi_{1}^{1} \sum_{i=0}^{\infty}\left\{f\left(X_{r(i)}\right)-f\left(X_{e(i)}\right)\right\}^{2} \\
& +2 \xi_{1}^{1} \sum_{n=-\infty}^{+\infty} 1_{D}\left(X_{n}\right) \varphi\left(X_{n}\right) N^{D} \varphi\left(X_{n}\right) \\
& -\xi_{1}^{1} \sum_{n=-\infty}^{+\infty} 1_{D}\left(X_{n}\right) \varphi^{2}\left(X_{n}\right) \\
& +2 \xi_{1}^{1} \sum_{i=0}^{\infty}\left\{f\left(X_{r(i)}\right)-f\left(X_{e(i)}\right)\right\} \sum_{n=e(i)}^{r(i)-1} \varphi\left(X_{n}\right) .
\end{aligned}
$$

Thus (3.16) can be rewritten

$$
\begin{aligned}
\xi_{1}^{1} \sum_{n=-\infty}^{+\infty} 1_{D}\left(X_{n}\right)\left\{f\left(X_{n+1}\right)-f\left(X_{n}\right)\right\}^{2} & \\
= & \xi_{1}^{1} \sum_{i=0}^{\infty}\left\{f\left(X_{r(i)}\right)-f\left(X_{e(i)}\right)\right\}^{2}+2 \mathcal{E}^{D}\left(f-H^{M} f, f-H^{M} f\right) \\
& +2 \xi_{1}^{1} \sum_{i=0}^{\infty}\left\{f\left(X_{r(i)}\right)-f\left(X_{e(i)}\right)\right\} \sum_{n=e(i)}^{r(i)-1} \varphi\left(X_{n}\right)
\end{aligned}
$$

On the other hand

$$
\begin{aligned}
\xi_{1}^{1} \sum_{i=0}^{\infty} & \left\{f\left(X_{e(i)}\right)-f\left(X_{e(i)-1}\right)\right\}^{2} \\
= & \xi_{1}^{1} \sum_{i=0}^{\infty}\left\{f\left(X_{r(i)}\right)-f\left(X_{e(i)-1}\right)\right\}^{2}-\xi_{1}^{1} \sum_{i=0}^{\infty}\left\{f\left(X_{r(i)}\right)-f\left(X_{e(i)}\right)\right\}^{2} \\
& -2 \xi_{1}^{1} \sum_{i=0}^{\infty}\left\{f\left(X_{e(i)}\right)-f\left(X_{e(i)-1}\right)\right\}\left\{f\left(X_{r(i)}\right)-f\left(X_{e(i)}\right)\right\} .
\end{aligned}
$$

Clearly

$$
\begin{aligned}
\mathfrak{\xi}_{1}^{1} \sum_{i=0}^{\infty}\left\{f\left(X_{e(i)}\right)-f\left(X_{e(i)-1}\right)\right\} & \left\{f\left(X_{r(i)}\right)-f\left(X_{e(i)}\right)\right\} \\
= & \xi_{1}^{1} \sum_{i=0}^{\infty}\left\{f\left(X_{e(i)}\right)-f\left(X_{e(i)-1}\right)\right\} \sum_{n=e(i)}^{r(i)-1} \varphi\left(X_{n}\right)
\end{aligned}
$$

and so (3.17) and (3.18) together with (vi) give 


$$
\begin{aligned}
\mathcal{E}^{D, r}(f, f)= & \mathcal{E}^{D}\left(f-H^{M} f, f-H^{M} f\right) \\
& +\frac{1}{2} \xi_{1}^{1} \sum_{i=0}^{\infty}\left\{f\left(X_{r(i)}\right)-f\left(X_{e(i)-1}\right)\right\}^{2} \\
& +\xi_{1}^{1} \sum_{i=0}^{\infty}\left\{f\left(X_{r(i)}\right)-f\left(X_{e(i)-1}\right)\right\} \sum_{n=e(i)}^{r(i)-1} \varphi\left(X_{n}\right)
\end{aligned}
$$

But the last term must vanish since it changes sign under time reversal and so (iv) is proved. Finally (ii) and (iii) follow from (iv) by polarization.

We turn now to some results of a global nature. An obvious application of (1.11) gives

$$
\mathcal{E}(f, f)=\frac{1}{2} \xi_{1}^{1} \sum_{n=-\infty}^{+\infty}\left\{f\left(X_{n+1}\right)-f\left(X_{n}\right)\right\}^{2} .
$$

Asymptotic behavior for $f$ in $\mathcal{F}^{\text {min }}$ is disposed of in

Theorem 3.3. For $f$ in the minimal Dirichlet space $\mathcal{F}^{\mathrm{min}}$,

$$
f\left(X_{n}\right) \rightarrow 0 \text { as } n \uparrow \infty
$$

[a.e. $\mathfrak{B}_{x}$ ] for all $x$ in $I$ and also [a.e. $\mathfrak{B}^{1}{ }_{1}$ ].

Proof. These results follow from transience when $f$ has finite support and follow in general from the estimate (2.6) together with Theorem 2.5, Theorem 2.10 and the estimate

$$
\begin{aligned}
\alpha(x) \mathfrak{B}_{x}\left[\sigma_{E}<\infty\right] & =\sum_{y} \alpha(x) N(x, y) L_{E}^{1}(y) \\
& =\sum_{y} \alpha(y) L_{E}^{1}(y) N(y, x) \\
& \leqq N(x, x) \operatorname{Cap}(E)
\end{aligned}
$$

with the computation valid when Cap $(E)<+\infty$ and therefore with the result valid for general $E$.

For harmonic $h$ in $\mathfrak{F}$ we have

Theorem 3.4. Let $h$ in $F$ be harmonic.

(i) $\ell^{h}(d y)=h^{\cdot}(y) \ell^{1}(d y)$ with $h^{\cdot}$ in $L^{2}\left(\ell^{1}\right)$

(ii) $h\left(X_{n}\right) \rightarrow h^{\cdot}\left(X_{\infty}\right)$ as $n \uparrow \infty$ [a.e. $\mathfrak{P}_{x}$ ] for $x$ in I and also [a.e. $\mathfrak{B}_{1}{ }^{1}$ ].

Proof. By (3.20)

$$
\mathcal{E}(h, h) \geqq \frac{1}{2} L_{\{0\}}^{1}(0) \xi_{0} \sum_{n=0}^{\infty}\left\{h\left(X_{n+1}\right)-h\left(X_{n}\right)\right\}^{2}
$$

and so $h\left(X_{n}\right), n \geqq 0$ is an $L^{2}$ bounded martingale relative to $\mathfrak{B}_{0}$. The theorem now follows from Theorem 1.8. 
3.1. Notation. For $h$ in $F$ harmonic, $h^{\cdot}$ is the unique function in $L^{2}\left(\ell^{1}\right)$ such that $\ell^{h}=h \cdot \ell^{1}$ or, equivalently,

$$
h(x)=\int K(x, y) h^{\circ}(y) \ell^{1}(d y) .
$$

In general we write

$$
H \varphi(x)=\int K(x, y) \varphi(y) \ell^{1}(d y)
$$

for $\varphi$ in $L^{1}\left(\ell^{1}\right)$ so that in particular $h=H h^{\circ}$ for $h$ in $\mathcal{F}$ harmonic. This use of the symbol $H$ is logically distinct from (2.8) but context will always make it clear which is meant.

3.2. Definition. The universal Dirichlet space on the boundary is the collection $\mathfrak{C}$ of $h^{\cdot}$ in $L^{2}\left(\ell^{1}\right)$ such that $H h^{\circ}$ belongs to the universal Dirichlet space $F$. The universal Dirichlet form on the boundary is

$$
\frac{1}{2} \alpha(0) \Theta\left\langle h^{\cdot}, h^{\cdot}\right\rangle=\frac{1}{2} \alpha(0) \int \ell^{1}(d y) \int \ell^{1}(d z) \Theta(y, z)\left\{h^{\cdot}(y)-h^{\cdot}(z)\right\}^{2} .
$$

We finish this section with

Theorem 3.5. If $h^{\circ}$ belongs to $\mathfrak{H C}$ then

$$
\begin{aligned}
\varepsilon\left(H h^{\cdot}, H h^{\cdot}\right) & =\frac{1}{2} \xi_{1}^{1}\left\{h^{\cdot}\left(X_{+\infty}\right)-h^{\cdot}\left(X_{-\infty}\right)\right\}^{2} \\
& =\frac{1}{2} \alpha(0) \Theta\left\langle h^{\cdot}, h^{\cdot}\right\rangle
\end{aligned}
$$

with the understanding that each of these expressions converges if and only if $h$. belongs to $\mathfrak{H C}$.

Proof. The first identity follows since

$$
\begin{aligned}
\frac{1}{2} \xi_{1}^{1}\left\{h^{\cdot}\left(X_{+\infty}\right)-h^{\cdot}\left(X_{-\infty}\right)\right\}^{2} & =\frac{1}{2}\left(\xi_{1}^{1} \sum_{n=-\infty}^{+\infty}\left\{H h^{\cdot}\left(X_{n+1}\right)-H h^{\cdot}\left(X_{n}\right)\right\}^{2}\right. \\
& =\varepsilon(H h, H h)
\end{aligned}
$$

by (3.20) and the second identity then follows from (1.24).

4. Continuous time Dirichlet spaces. For the reader's convenience we begin this section by collecting some general results on Dirichlet spaces. The basic ideas go back to Beurling and Deny [1] but the particular point of view taken here is that of M. Fukishima [10].

Let $X$ be a separable locally compact Hausdorff space and let $d x$ be a Radon measure on $X$. A symmetric submarkovian semigroup on $L^{2}(X)$ is a collection of operators $\boldsymbol{P}_{t}, t>0$ on $L^{2}(X)$ satisfying

4.1.1. Each $P_{t}$ is a symmetric contraction on $L^{2}(X)$. 
4.1.2. $\boldsymbol{P}_{t} \boldsymbol{P}_{\mathrm{s}}=\boldsymbol{P}_{t+s}$ and $\boldsymbol{P}_{t} \rightarrow 1$ in the strong operator topology as $t \downarrow 0$.

4.1.3. $0 \leqq P_{t} f \leqq 1$ whenever $0 \leqq f \leqq 1$.

Of course relations for functions in $L^{2}(X)$ are understood in the [a.e. dx] sense. The resolvent operators $G_{u}, u>0$ are defined by

$$
G_{u}=\int_{0}^{\infty} d t e^{-u t} \boldsymbol{P}_{t}
$$

and the generator $A$ is the unique nonpositive definite self adjoint operator on $L^{2}(X)$ such that

$$
G_{u}=(u 1-A)^{-1} \quad u>0 .
$$

Let $(-A)^{1 / 2}$ be the unique nonnegative definite square root of $-A$. The associated Dirichlet space is the pair $(F, E)$ where

$$
\begin{gathered}
F=\text { domain }(-A)^{1 / 2} \\
E(f, g)=\int d x(-A)^{1 / 2} f(x)(-A)^{1 / 2} g(x) \quad f, g \text { in } F
\end{gathered}
$$

An important tool for analyzing $(F, E)$ is the elementary

Theorem 4.1. (i) $f$ in $L^{2}(X)$ belongs to $F$ if and only if

$$
\sup _{t>0} \int d x(1 / t)\left\{f-P_{t} f\right\}(x) f(x)<+\infty
$$

and in this case

$$
E(f, f)=\operatorname{Lim} \int d x(1 / t)\left\{f-P_{t} f\right\}(x) f(x) \quad(t \downarrow 0) .
$$

(ii) $f$ in $L^{2}(X)$ belongs to $F$ if and only if

$$
\sup _{u>0} \int d x u\left\{f-u G_{u} f\right\}(x) f(x)<+\infty
$$

and in this case

$$
E(f, f)=\operatorname{Lim} u \int d x\left\{f-u G_{u} f\right\}(x) f(x) \quad(u \uparrow \infty) .
$$

Proof. By the spectral theorem there exists a measure space $\left(X^{*}, d x^{*}\right)$, an isometry $\mathcal{U}: L^{2}(X) \rightarrow L^{2}\left(X^{*}\right)$ and a negative measurable function $A^{*}$ on $X^{*}$ such that $f$ in $L^{2}(X)$ belongs to $F$ if and only if

$$
-\int d x^{*} A^{*}\left(x^{*}\right)\{u f\}^{2}\left(x^{*}\right)<+\infty
$$

and then 


$$
E(f, f)=-\int d x^{*} A^{*}\left(x^{*}\right)\{u f\}^{2}\left(x^{*}\right)
$$

Also

$$
\begin{aligned}
\int d x\left\{f-\boldsymbol{P}_{t} f\right\}(x) f(x) & =\int d x^{*}\left(1-e^{t A *(x)}\right)\{u f\}^{2}\left(x^{*}\right) \\
\int d x\left\{f-u G_{u} f\right\}(x) f(x) & =\int d x^{*}\left\{-A^{*}\left(x^{*}\right) /\left(u-A^{*}\left(x^{*}\right)\right)\right\}\{u f\}^{2}\left(x^{*}\right)
\end{aligned}
$$

and the theorem follows directly.

It is elementary that $F$ is a Hilbert space relative to any of the inner products

$$
E_{u}(f, g)=E(f, g)+u \int d x f(x) g(x) \quad u>0 .
$$

A deeper result can be established with the help of Theorem 4.1. We say that $g$ is a normalized contraction of $f$ if there exist everywhere defined versions of $f$ and $g$ such that

$$
|g(x)| \leqq|f(x)| ;|g(x)-g(y)| \leqq|f(x)-f(y)| .
$$

With our hypotheses on $X$, it is easy to show that for $t>0$ there exists a symmetric measure $\boldsymbol{P}_{t}(d x, d y)$ on $\boldsymbol{X} \times \boldsymbol{X}$ such that

$$
\int d x f(x) \boldsymbol{P}_{t} g(x)=\iint \boldsymbol{P}_{t}(d x, d y) f(x) g(x)
$$

for $f, g$ in $L^{2}(X)$. Now consider $f$ in $F$ and let $g$ be a normalized contraction of $f$. Then

$$
\begin{aligned}
\int d x\left\{g-\boldsymbol{P}_{t} g\right\}(x) & g(x) \\
& =\int d x g^{2}(x)\left\{1-\boldsymbol{P}_{t} 1\right\}(x)+\iint \boldsymbol{P}_{t}(d x, d y)\left\{g^{2}(x)-g(x) g(y)\right\} \\
& =\int d x g^{2}(x)\left\{1-\boldsymbol{P}_{t} 1\right\}(x)+\frac{1}{2} \iint \boldsymbol{P}_{t}(d x, d y)\{g(x)-g(y)\}^{2}
\end{aligned}
$$

and it follows that

$$
\int d x\left\{g-\boldsymbol{P}_{t} g\right\}(x) g(x) \leqq \int d x\left\{f-\boldsymbol{P}_{t} f\right\}(x) f(x) .
$$

Thus by Theorem $4.1 g$ belongs to $F$ and $E(g, g) \leqq E(f, f)$. Summarizing, the pair $(F, E)$ satisfies

4.2.1. $F$ is a Hilbert space relative to any of the inner products (4.2).

4.2.2. If $f$ is in $F$ and if $g$ is a normalized contraction of $f$, then also $g$ belongs to $F$ and $E(g, g) \leqq E(f, f)$. 
In general any pair $(F, E)$ with $F$ a dense linear subset of $L^{2}(X)$ and with $E$ a bilinear form on $F$ will be called a Dirichlet space on $L^{2}(X)$ if it satisfies 4.2.1 and 4.2.2. We have shown above that the Dirichlet space associated with a symmetric submarkovian resolvent on $L^{2}(X)$ is a Dirichlet space on $L^{2}(X)$. We now prove conversely that every Dirichlet space $(F, E)$ on $L^{2}(X)$ is associated with a unique symmetric submarkovian semigroup.

Let $G_{u}, u>0$ be the unique bounded operator from $L^{2}(X)$ into $F$ determined by

$$
E_{u}\left(G_{u} \varphi, g\right)=\int d x \varphi(x) g(x)
$$

for $\varphi$ in $L^{2}(X)$ and $g$ in $F$. It is easy to verify the resolvent identity

$$
G_{u}-G_{v}=(v-u) G_{u} G_{v} \quad u, v>0
$$

and that for $u>0$ the operator $u G_{u}$ is actually a symmetric contraction on $L^{2}(X)$. Thus by the Hille-Yosida theorem, or by the spectral theorem, there exists a strongly continuous symmetric contraction semigroup $\boldsymbol{P}_{t}, t>0$ on $L^{2}(X)$ with $G_{u}$ the resolvent operators. We will be done if we show that each $\boldsymbol{P}_{\boldsymbol{t}}$ satisfies

$$
\begin{aligned}
& \boldsymbol{P}_{t} \varphi \geqq 0 \quad \text { whenever } \varphi \geqq 0, \\
& \boldsymbol{P}_{t} \varphi \leqq 1 \quad \text { whenever } \varphi \leqq 1
\end{aligned}
$$

which will certainly follow if we show that

$$
T P_{t} \varphi=P_{t} \varphi \quad \text { whenever } T \varphi=\varphi
$$

where $T$ is any mapping from the reals to the reals satisfying

$$
T 0=0 ;|T \alpha-T \beta| \leqq|\alpha-\beta| .
$$

We establish instead the equivalent

$$
T u G_{u} \varphi=u G_{u} \varphi \quad \text { whenever } T \varphi=\varphi .
$$

For this purpose fix $\varphi$ in $L^{2}(X)$ satisfying $T \varphi=\varphi$ and for $f$ in $F$ and $u>0$ consider

$$
\begin{aligned}
E_{u}\left(f-u G_{u} \varphi, f-u G_{u} \varphi\right)= & E(f, f)+u \int d x\left\{f^{2}(x)+\varphi(x) u G_{u} \varphi(x)-2 f(x) \varphi(x)\right\} \\
= & E(f, f)+u \int d x\{f-\varphi\}^{2}(x) \\
& +u^{2} \int d x \varphi(x) G_{u} \varphi(x)-u \int d x \varphi^{2}(x) .
\end{aligned}
$$

It follows that the functional

$$
\Phi(f)=E(f, f)+u \int d x\{f-\varphi\}^{2}(x)
$$


has the unique minimum $f=u G_{u} \varphi$. But in general

$$
\Phi(T f)=E(T f, T f)+u \int d x\{T f-T \varphi\}^{2}(x) \leqq \Phi(f, f)
$$

by 4.2.2 and (4.7') follows.

Remark. The above arguments go through for more general measure spaces, but some restriction is needed to permit (4.4). Possibly a natural setting is standard measure spaces as defined by Parthasarathy in [15].

We return now to the state space $I$. The object of direct interest is a standard transition matrix $\boldsymbol{P}_{t}(x, y)$ on $I$. That is, $\boldsymbol{P}(x, y)$ is defined for $t>0$ and for $x, y$ in $I$ and satisfies

4.3.1. $\quad \boldsymbol{P}_{t}(x, y) \geqq 0$ and $\sum_{y} \boldsymbol{P}_{t}(x, y) \leqq 1$.

4.3.2. $\sum_{z} \boldsymbol{P}_{s}(x, z) \boldsymbol{P}_{t}(z, y)=\boldsymbol{P}_{s+t}(x, y)$.

4.3.3. $\boldsymbol{P}_{t}(x, y) \rightarrow \boldsymbol{\epsilon}_{x}(y)$ as $t \downarrow 0$.

As in earlier sections we are using $\epsilon_{x}$ to denote the point mass concentrated at $x$. Thus 4.3 .3 is equivalent to

$$
\boldsymbol{P}_{t}(x, x) \rightarrow 1 ; \boldsymbol{P}_{t}(x, y) \rightarrow 0 \text { for } x \neq y .
$$

To maintain a formal distinction between measures and functions we introduce a separate symbol $e_{x}$ for the indicator of the set $\{x\}$.

It follows from 4.3.3 (see [2]) that

$$
q(x)=\operatorname{Lim}(1 / t)\left\{1-\boldsymbol{P}_{t}(x, x)\right\} \quad(t \downarrow 0)
$$

exists for $x$ in $I$ with $0 \leqq q(x) \leqq+\infty$. In the terminology of [2] we assume that every state is stable and nonabsorbing. Thus

$$
0<q(x)<+\infty .
$$

Under these conditions the matrix elements $\boldsymbol{P}_{t}(x, y)$ are continuously differentiable for $t \geqq 0$. (Again see [2]. By a deeper result of Ornstein [14] or [2, p. 210] this is true for $x \neq y$ even without the restriction (4.9).) Thus there exists a substochastic matrix $P(x, y)$ vanishing on the diagonal such that

$$
(1 / t) \boldsymbol{P}_{t}(x, y) \rightarrow P(x, y) \quad(t \downarrow 0)
$$

for $x \neq y$. We assume $P$ is strictly stochastic:

$$
\sum_{y} P(x, y)=1
$$

We remark that (4.11) is automatic if $\boldsymbol{P}_{t}(x, y)$ is conservative:

$$
\sum_{\nu} \boldsymbol{P}_{t}(x, y)=1
$$

(See [2].) 
We restrict our attention now to transition matrices satisfying:

4.4 Condition of symmetry. There exists an everywhere positive measure $m(x)$ such that

$$
m(x) \boldsymbol{P}_{t}(x, y)=m(y) \boldsymbol{P}_{t}(y, x)
$$

Following Feller [7] we will generally refer to $P$ as the road map, to $q$ as the rate and to $m$ as the speed measure. Condition (4.12) yields the corresponding condition

$$
\alpha(x) P(x, y)=\alpha(y) P(y, x)
$$

for the road map with $\alpha$ the measure defined by

$$
\alpha(x)=m(x) q(x) .
$$

It will turn out that there is no loss of generality in assuming that $P$ is irreducible and transient (see the remark following Theorem 5.1) and we do so from now on. Thus $P$ satisfies the Basic Hypothesis 1.1 and Sections 1 through 3 are applicable.

We begin our analysis of $\boldsymbol{P}_{t}$ with

Theorem 4.2. The operators $\boldsymbol{P}_{t}, t>0$ form a symmetric submarkovian semigroup on $L^{2}(m)$.

Proof. Symmetry and the semigroup property are obvious. That each $\boldsymbol{P}_{\boldsymbol{t}}$ is a contraction on $L^{2}(m)$ follows from

$$
\sum m(x)\left\{\boldsymbol{P}_{t} f\right\}^{2}(x) \leqq \sum m(x) \boldsymbol{P}_{t} f^{2}(x) \leqq \sum m(x) f^{2}(x) .
$$

To check that $\boldsymbol{P}_{t} \rightarrow 1$ is the strong operator topology as $t \downarrow 0$ it suffices to consider the action on $e_{x}$ and since the $\boldsymbol{P}_{t}$ are contractions on $L^{\infty}$ it suffices to show that

But this follows since

$$
\sum_{y} m(y)\left|e_{x}(y)-\boldsymbol{P}_{t} e_{x}(y)\right| \rightarrow 0
$$

$$
\begin{aligned}
\sum_{\nu} m(y)\left|e_{x}(y)-\boldsymbol{P}_{t} e_{x}(y)\right| & =m(x)\left\{1-\boldsymbol{P}_{t}(x, x)\right\}+\sum_{y \neq x} m(y) \boldsymbol{P}_{t}(y, x) \\
& \leqq 2 m(x)\left\{1-\boldsymbol{P}_{t}(x, x)\right\} .
\end{aligned}
$$

Associated Dirichlet spaces are characterized in the following theorem which is itself an immediate consequence of Theorem 4.1-(i).

Theorem 4.3. Let $\boldsymbol{P}_{t}(x, y)$ be a standard transition matrix satisfying (4.8), (4.10) and (4.12) and let $(F, E)$ be the associated Dirichlet space. Then

$$
\begin{gathered}
{F^{\min }} \cap L^{2}(m) \quad \text { is contained in } F \\
E(f, g)=\mathcal{E}(f, g) \quad f, g \text { in } \mathcal{F}^{\min } \cap L^{2}(m) .
\end{gathered}
$$

Conversely if $(F, E)$ is a Dirichlet space on $L^{2}(m)$ satisfying (4.15), then it is associated with a unique standard transition matrix satisfying (4.8), (4.10) and (4.12). 
5. The minimal process. The simplest example of a Dirichlet space on $L^{2}(m)$ satisfying (4.15) is introduced in

5.1. Definition. The minimal Dirichlet space is the pair $\left(F^{\mathrm{min}}, \varepsilon\right)$ where

$$
F^{\text {min }}=\mathscr{F}^{\text {min }} \cap L^{2}(m) .
$$

Let $\boldsymbol{P}_{t}{ }^{\min }(x, y)$ be the well known minimal transition matrix of W. Feller [7]. This is defined by

$$
\begin{aligned}
\boldsymbol{P}_{t}^{(0)}(x, y) & =e^{-t q(x)} \boldsymbol{\epsilon}_{x}(y) \\
\boldsymbol{P}_{t}^{(n+1)} & =\int_{0}^{t} d s e^{-s \alpha} q P \boldsymbol{P}_{t-s}^{(n)} \\
\boldsymbol{P}_{t}^{\min } & =\sum_{n=0}^{\infty} \boldsymbol{P}_{t}^{(n)} .
\end{aligned}
$$

It is easy to check that the matrix elements $\boldsymbol{P}_{t}{ }^{\mathrm{min}}(x, y)$ form a transition matrix satisfying (4.8), (4.10) and (4.12). Also it is important that $\boldsymbol{P}_{t}{ }^{\mathrm{min}}$ can be characterized as the minimal nonnegative solution of the integral equation

$$
P_{t}^{\min }=e^{-t a} 1+\int_{0}^{t} d s q e^{-s a} P P_{t-s}^{\min }
$$

and also as the minimal nonnegative solution of

$$
P_{t}^{\min }=e^{-t a} 1+\int_{0}^{t} d s P_{s}^{\min } q e^{-(t-s) a} 1
$$

where 1 is understood to be the identity matrix in (5.2) and $\left(5.2^{\prime}\right)$. We begin with

Theorem 5.1. The minimal Dirichlet space $\left(F^{\mathrm{min}}, \mathcal{E}\right)$ is the Dirichlet space associated with the minimal transition matrix $\boldsymbol{P}_{t}{ }^{\min }(x, y)$.

Proof. Let $\left(\boldsymbol{F}^{0}, E^{0}\right)$ be the Dirichlet space associated with $\boldsymbol{P}_{t}{ }^{\text {min }}$. By (4.15), $F^{\text {min }}$ is contained in $F^{0}$ and $E^{0}(f, g)=\mathcal{E}(f, g)$ for $f, g$ in $F^{\text {min }}$. Thus it suffices to show that actually $\boldsymbol{F}^{0}$ is contained in $\boldsymbol{F}^{\mathrm{min}}$. To see this let $\boldsymbol{P}^{0}{ }_{t}$ be the transition matrix such that $\left(\boldsymbol{F}^{\mathrm{min}}, \varepsilon\right)$ is the associated Dirichlet space. Then $\boldsymbol{P}^{0}{ }_{t}$ satisfies (4.8) and (4.10) and it follows easily that $\boldsymbol{P}^{0}{ }_{t}$ satisfies (5.2). But $\boldsymbol{P}_{t}{ }^{\mathrm{min}}$ is the minimal solution of (5.2) and so $\boldsymbol{P}_{t}{ }^{\mathrm{min}} \leqq \boldsymbol{P}^{0}{ }_{t}$. But then it follows from Theorem 4.1 that $F^{0}$ is contained in $F^{\mathrm{min}}$ and we are done.

Remark. If the road map $P$ were recurrent, then clearly $\boldsymbol{P}_{t}{ }^{\min }$ would be conservative and therefore if $m$ were bounded the constant function 1 would belong to $F^{\mathrm{min}}$. From this it follows that the completion of $L^{2}(\alpha)$ with respect to $\mathcal{E}$ must contain 1 and therefore cannot be a Hilbert space.

We augment $I$ as in Section 1 by introducing a "dead point" $\partial$. The standard sample space $\Omega$ is the collection of mapping $\omega$ from the half line $[0, \infty)$ into the augmented state space $I \cup\{\partial\}$ which satisfy the following conditions. 
5.2.1. $\omega(t)$ is right continuous and has one sided limit everywhere.

5.2.2. There exists a life time $\zeta(\omega)$ with $0 \leqq \zeta(\omega) \leqq+\infty$ such that $\omega(t)=\partial$ if and only if $t \geqq \zeta(\omega)$ and such that $\omega(t-0) \neq \partial$ for $0<t<\zeta$.

We remark that the topology on $I \cup\{\partial\}$ is irrelevant, so long as the induced topology on $I$ is discrete.

As usual we introduce trajectory variables $X_{t}$ by

$$
X_{t}(\omega)=\omega(t) \text {. }
$$

It is well known that for each $x$ in $I$ there is a unique probability $\mathcal{P}_{x}{ }^{\text {min }}$ on $\Omega$ such that

$$
\begin{aligned}
P_{x}^{\min }\left[X_{0}=x, X_{t_{1}}=x_{1}, \cdots,\right. & \left.X_{t_{n}}=x_{n}\right] \\
& =\boldsymbol{P}_{t_{1}}^{\min }\left(x, x_{1}\right) \boldsymbol{P}_{t_{2}-t_{1}}^{\min }\left(x_{1}, x_{2}\right) \cdots \boldsymbol{P}_{t_{n}-t_{n-1}}^{\min }\left(x_{n-1}, x_{n}\right)
\end{aligned}
$$

for any choice of $0=t_{0}<t_{1}<\cdots<t_{n}$ and $x_{1}, \cdots, x_{n}$ in $I$. Indeed we have the following

5.3. Probabilistic description of $\mathcal{P}_{x}^{\min }$. There exists an increasing sequence of positive random variables $\left\{\tau_{n}\right\}_{0 \leqq n<\infty}$ and a sequence $\left\{Y_{n}\right\}_{0 \leqq n<\infty}$ of random points in $I$ such that

5.3.1. $\tau_{0}=0$ and $Y_{0}=x$.

5.3.2. The random variables $\left\{Y_{n}\right\}_{0 \leqq n<\infty}$ have the same distribution as the trajectory variables $\left\{X_{n}\right\}_{0 \leqq n<\infty}$ relative to the discrete time probability $P_{x}$ determined by (1.6).

5.3.3. Conditioned on the sequence $\left\{Y_{n}\right\}_{0 \leqq n<\infty}$, the increments $\left\{\tau_{n+1}-\right.$ $\left.\tau_{n}\right\}_{0 \leqq n<\infty}$ are mutually independent and $\tau_{n+1}-\tau_{n}$ is exponentially distributed at the rate $q\left(Y_{n}\right)$. That is,

$$
\boldsymbol{P}_{x}^{\min }\left[\tau_{n+1}-\tau_{n}>t \mid\left\{Y_{m}\right\}_{m=0}^{\infty}\right]=e^{-t Q\left(Y_{n}\right)} .
$$

5.3.4. $X_{t}=Y_{n}$ for $\tau_{n} \leqq t<\tau_{n+1}$.

5.3.5. $\tau_{n} \uparrow \zeta$ as $n \uparrow \infty$.

An easy way to verify all this is by an explicit construction as suggested by 5.3 .

Next we introduce some notation and machinery for the minimal process which are analogous to those introduced earlier for the discrete time process.

The potential operator $G^{\mathrm{min}}(x, y)$ is defined by

$$
G^{\mathrm{min}}(x, y)=\mathcal{E}_{x}^{\min } \int_{0}^{5} d t I\left[X_{t}=y\right]
$$

and it follows from 5.3 that

$$
G^{\mathrm{min}}(x, y) q(y)=N(x, y) .
$$


For $u>0$ the resolvent operator $G_{u}{ }^{\text {min }}$ is defined by

$$
G_{u}^{\mathrm{min}}(x, y)=\mathcal{E}_{x}^{\mathrm{min}} \int_{0}^{\zeta} d t e^{-u t} I\left(X_{t}=y\right)
$$

which is consistent with (4.1).

First hitting times and last exist times are defined as in Section 1:

$$
\begin{gathered}
\sigma(E)=\inf \left\{t \geqq 0: X_{t} \text { is in } E\right\} \\
\sigma^{*}(E)=\sup \left\{t>0: X_{t} \text { is in } E\right\}
\end{gathered}
$$

with the understanding that these are respectively $+\infty$ and $-\infty$ when not otherwise defined.

For $h>0$ and excessive the conditional minimal matrix is defined by

$$
P_{t}^{\min , h}(x, y)=(1 / h(x)) P_{t}^{\min }(x, y) h(y)
$$

and the conditioned minimal probability is the unique probability $\mathcal{P}_{x}^{\min , h}$ on $\Omega$ satisfying

$$
\begin{aligned}
\mathcal{P}_{x}^{\min , h}\left[X_{0}=x_{1} X_{1}=x_{1}, \cdots\right. & \left., X_{n}=x_{n}\right] \\
& =P_{t_{1}}^{\min , h}\left(x, x_{1}\right) P_{t_{2}-t_{1}}^{\min , h}\left(x_{1}, x_{2}\right) \cdots P_{t_{n}-t_{n-1}}^{\min , h}\left(x_{n-1}, x_{n}\right) .
\end{aligned}
$$

Using the appropriate analogue of 5.3 it is easy to see that

$$
X_{\zeta-0}=\operatorname{Lim} X_{t} \quad(t \uparrow \zeta)
$$

is [a.e. $\mathcal{P}_{x}^{\min , h}$ ] well defined as a point in $I \cup \Delta_{0}$.

The time reversal operator $\rho$ is defined on $\Omega \cap\left[X_{\zeta-0}\right.$ is in $\left.I\right]$ by

$$
\rho \omega(t)=\left\{\begin{array}{cr}
X_{\zeta-t-0} & 0 \leqq t<\zeta \\
\partial & t \geqq \zeta .
\end{array}\right.
$$

For $E$ a subset of $I$ the local time reversal operator $\rho_{E}$ and the truncation operator $\tau_{E}$ are defined on $\Omega \cap[\sigma(E)<+\infty] \cap\left[X_{\sigma^{*}(E)-0}\right.$ is in $\left.I\right]$ by

$$
\begin{aligned}
& \rho_{E} \omega(t)=\left\{\begin{array}{cr}
X_{\sigma^{*}(E)-t-0} & 0 \leqq t<\sigma_{E}^{*} \\
\partial & t \geqq \sigma_{E}^{*}
\end{array}\right. \\
& \tau_{E} \omega(t)=\left\{\begin{array}{lr}
X_{t} & 0 \leqq t<\sigma_{E}^{*} \\
\partial & t \geqq \sigma_{E}^{*}
\end{array}\right.
\end{aligned}
$$

Theorem 1.2 leads directly to

$$
\begin{aligned}
\sum_{x} \alpha(x) h^{\prime}(x) L^{h^{\prime}}(x) h(x) & \mathcal{E}_{x}^{\mathrm{min}, h}\left[X_{\zeta-0} \text { is in } I ; \xi \circ \rho\right] \\
= & \sum_{y} \alpha(y) h(y) L^{h}(y) h^{\prime}(y) \mathcal{E}_{y}^{\min , h^{\prime}}\left[X_{\zeta-0} \text { is in } I ; \xi\right]
\end{aligned}
$$




$$
\begin{aligned}
& \sum_{x} \alpha(x) h^{\prime}(x) L_{E^{\prime}}^{h^{\prime}}(x) h(x) \varepsilon_{x}^{\min , h} {\left[\sigma(E)<+\infty ; X_{\sigma^{*}\left(E^{\prime}\right)-0} \text { is in } I ; \xi \circ \tau_{E^{\prime}}\right] } \\
&= \sum_{\nu} \alpha(y) h(y) L_{E}^{h}(y) h^{\prime}(y) \varepsilon_{\nu}^{\min , h^{\prime}} \\
& \cdot\left[\sigma\left(E^{\prime}\right)<+\infty ; X_{\sigma^{*}\left(E^{\prime}\right)-0} \text { is in } I ; \xi \circ \tau_{E^{\prime}}\right]
\end{aligned}
$$

and in particular for $x, y$ in $I$,

$$
\begin{aligned}
& \alpha(x) h^{\prime}(x) L^{h^{\prime}}(x) h(x){P_{x}^{\min , h}}\left[X_{\zeta-0}=y\right] \\
& =\alpha(y) h(y) L^{h}(y) h^{\prime}(y) \mathcal{P}_{y}^{\min , h^{\prime}}\left[X_{\zeta-0}=x\right], \\
& \alpha(x) h^{\prime}(x) L_{E^{\prime}}^{h^{\prime}}(x) h(x) \mathcal{P}_{x}^{\min , h}\left[\sigma(E)<+\infty ; X_{\sigma^{*}(E)-0}=y\right] \\
& =\alpha(y) h(y) L_{E}^{h}(y) h^{\prime}(y) \mathcal{P}_{y}^{\min , h^{\prime}}\left[\sigma\left(E^{\prime}\right)<+\infty ; X_{\sigma^{*}\left(E^{\prime}\right)-0}=y\right],
\end{aligned}
$$

where $h, h^{\prime}>0$ are excessive and $E, E^{\prime}$ are subsets of $I$.

The subsets $\Omega_{k}$ and the extended sample space $\Omega_{\infty}$ are defined as in Section 1 for discrete time. For $h, h^{\prime}>0$ excessive there exists a unique measure $\rho^{\mathrm{min}, h}$ on $\Omega_{\infty}$ such that

$$
\mathcal{E}_{h^{\prime}}^{\min , h_{\xi}} \xi \circ J_{\infty, k}=\sum_{x} \alpha(x) h^{\prime}(x) L_{k}^{h^{\prime}}(x) h(x) \varepsilon_{x}^{\min , h_{\xi}} \xi
$$

for $\xi \geqq 0$ on $\Omega_{k}$ and vanishing on the dead trajectory $\delta_{k}$. The proof is formally the same as for discrete time but depends on two additional technical tools: the Skorohod topology for $\Omega$ [15, Chap. V] and the existence theorem [15, p. 139]. See [18] for details. (Apparently such an extension of Hunt's construction was first obtained by M. Weil [19].) Again we denote by $\Phi$ the natural mapping from $\Omega \cap\left[X_{0} \neq \partial\right]$ to $\Omega_{\infty}$ and note the identity

$$
h(x) \varepsilon_{x}^{\min , h} \xi \circ \Phi=\varepsilon_{G \min (\cdot, x) / m(x)}^{\min , h}
$$

for $\xi \geqq 0$ on $\Omega_{\infty}$. The trajectory variables $X_{t}$ and the time reversal $\rho$ are defined as for discrete time and then (5.6) leads to

$$
\mathcal{E}_{h^{\prime}}^{\min , h^{\prime}} \xi \circ \rho=\varepsilon_{h}^{\min , h^{\prime}} \xi
$$

with the special case

$$
\sum \nu(x) h(x) \varepsilon_{x}^{\min , h} \xi \circ \rho \circ \Phi=\varepsilon_{h}^{\min , G^{\min (\nu / m)}} \xi
$$

for $\xi \geqq 0$ and $\Omega_{\infty}$.

Fix a finite subset $D$ of $I$ and let $M=I-D$. The killed potential operator is defined by

$$
G^{\min , D}(x, y)=\mathcal{E}_{x}^{\min } \int_{0}^{\sigma(M)} d t I\left(X_{t}=y\right)
$$

and for $u>0$ the killed resolvent is defined by

$$
G_{u}^{\min , D}(x, y)=\varepsilon_{x}^{\min } \int_{0}^{\sigma(M)} d t e^{-u t} I\left(X_{t}=y\right) .
$$


Clearly

$$
G^{\min , D}(x, y) q(y)=N^{D}(x, y) .
$$

The hitting probabilities are the same as for discrete time:

$$
H^{M}(x, y)=\mathcal{P}_{x}^{\min }\left[X_{\sigma(M)}=y\right] .
$$

Also we introduce for $u>0$ the modified hitting probabilities

$$
H_{u}^{M}(x, y)=\varepsilon_{x}^{\min } e^{-u \sigma(M)} I\left(X_{\sigma(M)}=y\right) .
$$

Dual to these are the balayage operators

$$
\begin{aligned}
& \pi^{M}(y, x)=m(x) H^{M}(x, y)(1 / m(y)) \\
& \pi_{u}^{M}(y, x)=m(x) H_{u}^{M}(x, y)(1 / m(y)) .
\end{aligned}
$$

An important role will be played by the Feller kernels

$$
U_{u, v}^{M}(y, z)=\sum_{x \text { in } D} m(x) H_{u}^{M}(x, y)(1 / m(y)) H_{v}^{M}(x, z)(1 / m(z))
$$

defined for $0 \leqq u<v$ and for $y, z$ in $M$. For typographical convenience we identify $H^{M}{ }_{0}$ with $H^{M}$ and $\pi^{M}{ }_{0}$ with $\pi^{M}$. The following identities can be verified by routine computations.

$$
\begin{gathered}
H_{u}^{M}=H_{v}^{M}+(v-u) G_{u}^{D} H_{v}^{M} . \\
\pi_{u}^{M}=\pi_{v}^{M}+(v-u) \pi_{u}^{M} G_{v}^{D} . \\
U_{u, v}^{M}+(v-u) \varphi=(v-u) \pi_{u}^{M} H_{v}^{M} \varphi=(v-u) \pi_{v}^{M} H_{u}^{M} \varphi . \\
U_{u, w}^{M}=U_{u, v}^{M}+U_{v, w}^{M} \quad 0 \leqq u<v<w . \\
U_{u, v}^{M}(y, z)=U_{u, v}^{M}(z, y) .
\end{gathered}
$$

We will be particularly interested in the bilinear forms

$$
\begin{aligned}
& U_{u, v}^{M}(\varphi, \varphi)=\sum_{v, z \text { in } M} m(y) m(z) U_{u, v}^{M}(y, z) \varphi(y) \varphi(z) \\
& U_{u, v}^{M}\langle\varphi, \varphi\rangle=\sum_{y, z \text { in } M} m(y) m(z) U_{u, v}^{M}(y, z)\{\varphi(y)-\varphi(z)\}^{2}
\end{aligned}
$$

and the relation

$$
U_{u, v}^{M}\left(1, \varphi^{2}\right)=\frac{1}{2} U_{u, v}^{M}\langle\varphi, \varphi\rangle+\frac{1}{2} U_{u, v}^{M}(\varphi, \varphi)
$$

which follows from (5.13). It follows from (5.12) that

$$
U_{u, \infty}^{M}(y, z)=\operatorname{Lim} U_{u, v}^{M}(y, z) \quad(v \uparrow \infty)
$$

is well defined for $u \geqq 0$, although conceivably infinite. In fact (5.15) is finite. In order to show this and at the same time obtain an important formula involving these matrices we argue as in Section 3. 
Note first that for $u \geqq 0$

$$
\begin{gathered}
m(x) G_{u}^{\min , D}(x, y)=m(y) G_{u}^{\min , D}(y, x) \\
G_{u}^{\min }(x, y)=G_{u}^{\min , D}(x, y)+H_{u}^{M} G_{u}^{\min }(x, y)
\end{gathered}
$$

and therefore

$$
m(x) H_{u}^{M} G_{u}^{\mathrm{min}}(x, y)=m(y) H_{u}^{M} G_{u}^{\mathrm{min}}(y, x)
$$

or equivalently

$$
H_{u}^{M} G_{u}^{\mathrm{min}}(x, y)=G_{u}^{\mathrm{min}} \pi_{u}^{M}(x, y) .
$$

The appropriate analogues of Lemma 3.1 are

$$
E_{x}^{\min }\left[X\left(\sigma_{M}-0\right)=w ; X\left(\sigma_{M}\right)=y ; e^{-u \sigma(M)}\right]=G_{u}^{\min , D}(x, w) q(w) P(w, y)
$$

and therefore

$$
H_{u}^{M}(x, y)=\sum_{w \text { in } D} G_{u}^{\min , D}(x, w) q(w) P(w, y)
$$

for $x$ in $D$ and $q$ in $M$ and in particular

$$
u G_{u}^{D} 1+G_{u}^{D}\left(q P 1_{M}\right)=1
$$

on $D$.

Again we consider entrance and return times for excursions into $D$ :

$$
\begin{aligned}
& e(1)=\sigma(D) \\
& r(1)=\inf \left\{t>\sigma(D): X_{t} \text { is in } M\right\} . \\
& e(2)=\inf \left\{t>r_{1}: X_{t} \text { is in } D\right\}
\end{aligned}
$$

etc.,

with the understanding that $e(i), r(i)=+\infty$ when not otherwise defined. For $0 \leqq u<v$ and for $\varphi \geqq 0$ on $M \times M$ consider

$$
\begin{array}{rl}
\sum_{y, z \text { in } M} & m(y) m(z) U_{u, v}^{M}(y, z) \varphi(y, z) \\
= & (v-u) \varepsilon_{1}^{\min , 1} \sum_{i=0}^{\infty} \int_{e(i)}^{r(i)} d t \sum_{y} \sum_{z} H_{u}^{M}\left(X_{t}, y\right) H_{v}^{M}\left(X_{t}, z\right) \varphi(y, z) \\
= & (v-u) \varepsilon_{1}^{\min , 1} \sum_{i=0}^{\infty} \int_{e(i)}^{r(i)} d t \sum_{\nu} H_{u}^{M}\left(X_{t}, y\right) e^{-v[r(i)-t]} \varphi\left(y, X_{r(i)}\right) \\
= & (v-u) \varepsilon_{1}^{\min , 1} \sum_{i=0}^{\infty} \int_{e(i)}^{r(0)} d t e^{-u[r(i)-1]} e^{-v[t-e(i)]} \varphi\left(X_{r(i)}, X_{e(i)-0}\right)
\end{array}
$$

But

$$
\begin{aligned}
(v-u) \int_{e(i)}^{r(i)} d t e^{-u[r(i)-t]} e^{-v[t-e(i)]} & =e^{-u r(i)+v e(i)}\left\{e^{-(v-u) e(i)}-e^{-(v-u) r(i)}\right\} \\
& =e^{-u[r(i)-\theta(i)]}-e^{-v[r(i)-e(i)]}
\end{aligned}
$$


which increases to

$$
e^{-u[r(i)-e(i)]}
$$

as $v \uparrow \infty$. Thus

$$
\begin{aligned}
& \sum_{\nu, z \text { in } M} m(y) m(z) U_{u, \infty}^{M}(y, z) \varphi(y, z) \\
&=\mathcal{E}_{1}^{\min , 1} \sum_{i=0}^{\infty} e^{-u[r(i)-e(i)]} \varphi\left(X_{r(i)}, X_{e(i)-0}\right)
\end{aligned}
$$

for $\varphi \geqq 0$ on $M \times M$. But the right side of (5.22)

$$
\begin{aligned}
& =\mathcal{E}_{1}^{\min , 1} \sum_{i=0}^{\infty} \sum_{y} H_{u}^{M}\left(X_{e(i)}, y\right) \varphi\left(y, X_{e(i)-0}\right) \\
& =\varepsilon_{1}^{\min , 1} \sum_{i=0}^{\infty} \sum_{v} H_{u}^{M}\left(X_{r(i)-0}, y\right) \varphi\left(y, X_{r(i)}\right) \\
& =\varepsilon_{1}^{\min } \sum_{i=0}^{\infty} \int G^{D}\left[X\left(e_{i}\right), w\right] q(w) \sum_{\nu} H_{u}^{M}(w, y) \sum_{z} P(w, z) F(y, z) \\
& =\sum_{x, w \text { in } D} \sum_{y, z \text { in } M} \alpha(x) P 1_{M}(x) G^{D}(x, w) q(w) H_{u}^{M}(w, y) P(w, z) F(y, z) \\
& =\sum_{w \text { in } D} \sum_{y, z \text { in } M} m(w) H_{u}^{M}(w, y) q(w) P(w, z) F(y, z) .
\end{aligned}
$$

Thus

$$
U_{u, \infty}^{M}(y, z) m(y) m(z)=\sum_{x \text { in } D} m(x) H_{u}^{M}(x, y) q(x) P(x, z)
$$

and in particular

$$
U_{0, \infty}^{M}(y, z) m(y) m(z)=\Theta^{M}(y, z) \alpha(y) \alpha(z) .
$$

This identification of the Feller and Naïm kernel has been established is the context of Brownian motion by M. Fuskushima [8].

We turn now to corresponding global formulae. It follows from the probabilistic description 5.3 that the terminal position

$$
X_{\zeta-0}=\operatorname{Lim} X_{t} \quad(t \uparrow \zeta)
$$

is well defined [a.e. $\left.\mathcal{P}_{x}^{\mathrm{min}}\right]$ as a point in the extremal Martin boundary $\Delta_{0}$ and that for $\varphi$ in $L^{1}\left(l^{1}\right)$

$$
H \varphi(x)=\mathcal{E}_{x}^{\min } \varphi\left(X_{\zeta-0}\right)
$$

with $H$ defined by (3.22). For $u>0$ the modified global hitting operator $H_{u}$ is defined by

$$
H_{u} \varphi(x)=\varepsilon_{x}^{\min } e^{-u \zeta} \varphi\left(X_{\zeta-0}\right) .
$$

For $y$ in $\Delta_{0}$ and for $u>0$ let

$$
K_{u}(x, y)=K(x, y) \varepsilon_{x}^{K(\cdot, y)} e^{-u \zeta} .
$$


It is easy to check that

$$
K(x, y)=K_{u}(x, y)+u G_{u}^{\min } K(\cdot, y)(x)
$$

for $u>0$ and $y$ in $\Delta_{0}$. Also for $\varphi$ in $L^{1}\left(l^{1}\right)$

$$
\begin{aligned}
H_{u} \varphi(x) & =\int K_{u}(x, y) \varphi(y) \ell^{\mathrm{l}}(d y) \\
H_{\varphi} & =H_{u} \varphi+u G_{u}^{\mathrm{min}} H \varphi .
\end{aligned}
$$

A prequisite for introducing the active and passive boundary of W. Feller is

Lemma 5.2. For $y$ in the extremal Martin boundary $\Delta_{0}$

either $\quad \mathcal{P}_{x}^{\min , K(\cdot, y)}[\zeta<+\infty]=1$ for all $x$ in $I$

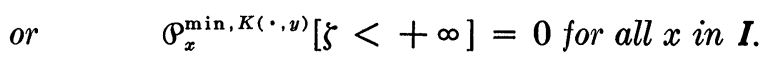

Proof. It suffices first to observe that

$$
\left.h(x)=K(x, y){P_{x}^{\min , K(\cdot, y)}}^{\zeta}=+\infty\right]
$$

is a harmonic function dominated by $K(\cdot, y)$ and therefore must be a constant multiple of $K(\cdot, y)$ and then to consider asymptotic behavior $\left[\right.$ a.e. $\mathcal{P}_{x}^{\min , K(\cdot, v)}$ ] of $h\left(X_{t}\right) / K\left(X_{t}, y\right)$ as $t \uparrow \zeta$.

5.4. Definition. $y$ in the extremal Martin boundary is active if

and passive if

$$
\odot_{x}^{\min , K(\cdot, y)}[\zeta<\infty]=1 \quad x \text { in } I
$$

$$
\mathcal{P}_{x}^{\min , K(\cdot, y)}[\zeta<\infty]=0 \quad x \text { in } I .
$$

The active part of the extremal Martin boundary $\Delta_{0}$ will be denoted by $\Delta_{m}$. The balayaged measure on $\Delta_{m}$ is defined by

$$
\pi_{1} m(d y)=\sum_{x} m(x) K_{1}(x, y) \ell^{1}(d y) .
$$

In general $\pi_{1} m$ need not be finite or even $\sigma$-finite and so $d \ell / d \pi_{1} m$ need not exist in the usual sense. However we can always define $d \ell^{1} / d \pi_{1} m$ to be the unique function in $L^{2}\left(\pi_{1} m\right)$ such that

$$
\int \pi_{1} m(d y)\left(d \ell^{1} / d \pi_{1} m\right)(y) \varphi(y)=\int \ell^{1}(d y) \varphi(y)
$$

for $\varphi$ in $L^{2}\left(\Delta_{m}, \pi_{1} m\right)$. Then for $u \geqq 0$ the global balayage operatore $\pi_{u}$ is defined by

$$
\pi_{u} f(y)=\sum m(x) f(x) K_{u}(x, y)\left(d \ell^{1} / d \pi_{1} m\right)(y)
$$

when it converges for $f$ on $I$. The analogues of $(5.10)$ and $\left(5.10^{\prime}\right)$ are

$$
\begin{aligned}
H_{u} \varphi & =H_{v} \varphi+(v-u) G_{u}^{\mathrm{min}} H_{v} \varphi \\
\pi_{u} f & =\pi_{v} f+(v-u) \pi_{v} G_{u}^{\mathrm{min}} f
\end{aligned}
$$


and are valid for $u, v \geqq 0$ with the understanding that $\varphi$ in (5.32) vanishes on the passive boundary $\Delta_{0}-\Delta_{m}$. Now it is easy to check that for $u>0$ the operator $H_{u}$ is bounded from $L^{2}\left(\Delta_{m}, \pi_{1} m\right)$ to $L^{2}(I, m)$, that $\pi_{u}$ is bounded from $L^{2}(I, m)$ to $L^{2}\left(\Delta_{m}, \pi_{1} m\right)$ and that

$$
\sum m(x) f(x) H_{u} \varphi(x)=\int \pi_{1} m(d y) \pi_{u} f(y) \varphi(y)
$$

for $f$ in $L^{2}(I, m)$ and for $\varphi$ in $L^{2}\left(\Delta_{m}, \pi_{1} m\right)$. For $0 \leqq u<v$ the global Feller kernel $U_{u, v}(y, z)$ is defined by

$$
U_{u, v}(y, z)=(v-u) \sum m(x) K_{u}(x, y) K_{v}(x, z) .
$$

The Feller operator $U_{u, v}$ is defined when it converges by

$$
U_{u, v} \varphi(y)=\int U_{u, v}(y, z) \varphi(z) \ell^{1}(d y)
$$

for $\varphi$ defined on $\Delta_{m}$ and for $y$ in $\Delta_{m}$. The appropriate analogues of (5.11) through (5.13) are

$$
\begin{gathered}
U_{u, w}=U_{u, v}+U_{v, w} \quad 0 \leqq u<v<w \\
U_{u, v}(y, z)=U_{u, v}(z, y) \\
\left(d \ell^{1} / d \pi_{1} m\right) U_{u, v} \varphi=(v-u) \pi_{u} H_{v} \varphi \\
=(v-u) \pi_{v} H_{u} \varphi
\end{gathered}
$$

again with the understanding in (5.37) that $\varphi$ vanishes on the passive boundary. As in (5.15) we use (5.35) to define

$$
U_{u, \infty}(y, z)=\operatorname{Lim} U_{u, v}(y, z) \quad(v \uparrow \infty) .
$$

The important bilinear forms are

$$
\begin{aligned}
& U_{u, v}(\varphi, \varphi)=\int \ell^{1}(d y) \int \ell^{1}(d z) U_{u, v}(y, z) \varphi(y) \varphi(z) \\
& U_{u, v}\langle\varphi, \varphi\rangle=\int \ell(d y) \int \ell^{1}(d z) U_{u, v}(y, z)\{\varphi(y)-\varphi(z)\}^{2}
\end{aligned}
$$

and again we note the relation

$$
U_{u, v}\left(1, \varphi^{2}\right)=\frac{1}{2} U_{u, v}\left\langle\varphi, \varphi>+\frac{1}{2} U_{u, v}(\varphi, \varphi)\right.
$$

which is valid when the left side converges absolutely.

A calculation exactly analogous to (5.21) establishes for $u>0$

$$
\int \ell^{1}(d y) \int \ell^{1}(d z) U_{u, \infty}(y, z) \varphi(y, z)=\varepsilon_{1}^{\min , 1} \varphi\left(X_{\zeta-0}, X_{\zeta^{*}}\right) e^{-u\left(\zeta-\zeta^{*}\right)}
$$

for $\varphi \geqq 0$ on $\Delta_{0} \times \Delta_{0}$. From this and (1.24) it follows that

$$
U_{0, \infty}(y, z)=\alpha(0) \Theta(y, z)
$$


[a.e. $\left.\ell^{1}(d y) \times \ell^{1}(d z)\right]$ on $\Delta_{m} \times \Delta_{m}$. (No doubt this identity can be shown to hold identically on $\Delta_{m} \times \Delta_{m}$. Again we refer to [8] where such an identity is established for Brownian motion.)

To obtain an analogous identity involving the passive part of the boundary, we introduce by analogy with (5.16) in [17] the "passive part of 1"

$$
p(x)=\odot_{x}^{\min }[\zeta=+\infty] .
$$

For $u>0$ and $\varphi \geqq 0$ on $\Delta_{m}$

$$
\begin{aligned}
\sum_{x} m(x) \int \ell^{1}(d y) p(x) u K_{u}(x, y) \varphi(y) \\
=\varepsilon_{1}^{\min , 1} \int_{\zeta^{*}}^{\zeta} d t p\left(x_{t}\right) u e^{-u(\zeta-t)} \varphi\left(X_{\zeta-0}\right) \\
=\varepsilon_{1}^{\min , 1} \int_{\zeta^{*}}^{\zeta} d t p\left(X_{t}\right) u e^{-u\left(t-\zeta^{*}\right)} \varphi\left(X_{\zeta^{*}}\right) \\
=\varepsilon_{1}^{\min , 1} \int_{\zeta^{*}}^{\zeta} d t u e^{-u\left(t-\zeta^{*}\right)} \varphi\left(X_{\zeta^{*}}\right) I(\zeta=+\infty) \\
=\varepsilon_{1}^{\min , 1} I\left(-\infty<\zeta^{*}<\zeta=+\infty\right) \varphi\left(X_{\zeta *}\right) \\
=E_{1}^{\min , 1} I\left(-\infty=\zeta^{*}<\zeta<+\infty\right) \varphi\left(X_{\zeta-0}\right)
\end{aligned}
$$

and there follows for $u>0$

$$
\sum m(x) p(x) u K_{u}(x, y)=\alpha(0) \int_{\Delta_{0}-\Delta_{m}} \Theta(y, z) \ell^{1}(d z)
$$

or equivalently

$$
u \pi_{u} p(y)=\left(d \ell^{1} / d \pi_{1} m\right)(y) \alpha(0) \int_{\Delta_{0}-\Delta_{m}} \Theta(y, z) \ell^{1}(d z)
$$

[a.e. $\left.\ell^{1}(d y)\right]$ on the active boundary $\Delta_{m}$.

It will be convenient in Section 6 to regard $H$ and $H_{u}$ also as operators on the universal Dirichlet space $\mathcal{F}$. The action of $H$ is defined by (2.8). We define $H_{u}$ by

$$
H_{u} f=H f-u G_{u}^{\min } f .
$$

It is easy to check that if $h=H f$ and if $h$ is defined by 3.1 , then

$$
\begin{gathered}
H f=H h^{\circ} \\
H_{u} f=H_{u} h .
\end{gathered}
$$

6. Classification. We begin by considering a general standard transition matrix $\boldsymbol{P}_{t}(x, y)$ satisfying (4.8), (4.10) and (4.12) together with the corresponding Dirichlet space $(F, E)$. In order to obtain a decent process we compactify the state space $I$ as in the appendix of [17]. 
Let $\boldsymbol{B}_{0}$ be a subset of $\boldsymbol{F} \cap L^{1}(m) \cap L^{\infty}(m)$ satisfying

6.1.1. $B_{0}$ is an algebra and contains all functions with finite support.

6.1.2. $\boldsymbol{B}_{0}$ is dense in $F$ relative to the equivalent inner products $E_{u}, u>0$.

6.1.3. The uniform closure $\boldsymbol{B}$ of $\boldsymbol{B}_{0}$ is separable.

Clearly $\boldsymbol{B}$ is a commutative Banach algebra. Its maximal ideal space $\boldsymbol{X}$ is the desired compactification of $\boldsymbol{I}$. This is a separable locally compact Hausdorff space which is compact if and only if the function 1 belongs to $B$. The original state space $I$ is densely imbedded in $X$ and the induced topology on $I$ is the discrete topology. The dead point $\partial$ is adjoined to $X$ as the "point at infinity" when $X$ is not compact and as an isolated point when it is. All structures on $I$ are carried over to $X$ in the obvious way. The point of this is that $(F, E)$ is a regular Dirichlet space on $L^{2}(X, m)$ and therefore the results of [17] are directly applicable.

The standard sample space $\Omega$ is defined as for the minimal transition matrix except that $I$ is replaced by $X$. By the results of Section 2 in [17] there exists an exceptional set $N$ of $X$ which is "negligible" in an appropriate sense (and in particular does not intersect $I$ ) and a family of probabilities $\mathcal{P}_{x}$ indexed by $x$ in the complement $X-N$ which form a strong Markov process taking values in $X-N$ and having the usual regularity properties. That is, the process is quasi-left-continuous and the trajectories are right continuous with left hand limits existing everywhere.

Let $D$ be a finite subset of $I$ and as usual let $M=I-D$. The killed transition matrix $\boldsymbol{P}^{D}{ }_{t}(x, y)$ and the killed resolvent operators $G^{D}{ }_{u}$ are defined as for the minimal process. It follows from [17, Theorem 3.6] that the corresponding killed Dirichlet space $\left(F^{D}, E^{D}\right)$ is determined by

$$
\begin{gathered}
F^{D}=\left\{f \text { in } F: f^{*}=0 \text { q.e. on } X-D\right\} \\
E^{D}(f, g)=E(f, g) \quad f, g \text { in } F^{D}
\end{gathered}
$$

where $f^{*}$ is understood to be a quasi-continuous version of $f$ and where q.e. means quasi-everywhere in the terminology of [17, Section 1]. But $D$ is closed in $X$ and $m(X-I)=0$ and so it follows from [17, Lemma 1.15] that $f^{*}=0$ q.e. on $X-D$ if and only if $f=0$ on $M=I-D$. From this and Theorem 4.3 it follows that actually

$$
\left(F^{D}, E^{D}\right)=\left(F^{\min , D} \varepsilon^{D}\right)
$$

and therefore

$$
G_{u}^{D}=G_{u}^{\min , D} ; \quad P_{t}^{D}=P_{t}^{\min , D} .
$$

From this it follows easily that the process introduced above agrees with the minimal process up to the first exit from $I$. In particular

$$
H_{u}^{M}(x, y)=\mathcal{E}_{x}\left[e^{-u \sigma(M)} ; X_{\sigma(M)}=y\right]
$$


for $x$ in $D, y$ in $M$ and $X_{\sigma(\mathbf{x}-I)-0}$ is well defined [a.e. $\mathcal{P}_{x}$ ] as a point in the extremal Martin boundary $\Delta_{0}$ and has the same distribution as $X_{\zeta-0}$ relative to $\mathcal{P}_{x}{ }^{\text {min }}$ for $x$ in $I$.

It is also proved in [17, Theorem 3.6] that for $u>0$ the operator $H^{M}{ }_{u}$ is orthogonal projection relative to $E_{u}$ of $F$ onto the orthogonal complement of $F^{D}$ in $F$.

In order to apply the techniques of Section 5 in [17] we introduce the elementary additive functional

$$
A^{M}(t)=\int_{0}^{t} d s 1_{M}\left(X_{s}\right)
$$

the inverse functional

$$
B^{M}(s)=\inf \left\{t>0: A^{M}(t)>s\right\}
$$

and the time changed process

$$
X^{M}(t)=X\left[B^{M}(t)\right]
$$

Roughly speaking, $X^{M}{ }_{t}$ is defined by looking at $X_{t}$ only when it is in the complement $X-D$. Since $m$ does not charge $X-I$, nothing is changed if we replace $M$ by the full complement $X-D$ in (6.4). Our attention will focus on the time changed resolvents $R_{a}{ }^{M}$ defined by

$$
R_{a}^{M} \varphi(x)=\varepsilon_{x} \int_{0}^{\infty} d t e^{-a t} \varphi\left(X_{t}^{M}\right)
$$

which after an elementary change of variables can also be written

$$
R_{a}^{M} \varphi(x)=\mathcal{E}_{x} \int_{0}^{\infty} d t 1_{M}\left(X_{t}\right) e^{-a A^{M}(t)} \varphi\left(X_{t}\right) .
$$

We will also be interested in the modified resolvents

$$
R_{(u) a}^{M} \varphi(x)=\mathcal{E}_{x} \int_{0}^{\infty} d t 1_{M}\left(X_{t}\right) e^{-u t} e^{-a A^{M}(t)} \varphi\left(X_{t}\right)
$$

With the help of time reversal and a certain universality principle, it was proved in [17, Lemma 5.1] that the operators $R_{a}, a>0$ and for $u>0$ the operators $R_{(u) a}, a>0$ form symmetric resolvents relative to $L^{2}(M, m)$. The associated Dirichlet spaces are identified in

Theorem 6.1. (i) For $u>0$ the Dirichlet space associated with $\left\{R_{(u) a}^{M}, a>0\right\}$ is $\left(H^{M}, Q^{M}{ }_{(u)}\right)$ where

$$
\begin{gathered}
H^{M}=F \text { restricted to } M \\
Q_{(u)}^{M}(\varphi, \varphi)=E_{u}\left(H_{u}^{M} \varphi, H_{u}^{M} \varphi\right) .
\end{gathered}
$$

(ii) For $0<u<v$ 


$$
Q_{(v)}^{M}(\varphi, \varphi)=Q_{(u)}^{M}(\varphi, \varphi)+U_{u, v}^{M}(\varphi, \varphi)+(v-u) \sum_{\nu \text { in } M} m(y) \varphi^{2}(y)
$$

and therefore

$$
Q^{M}(\varphi, \varphi)=Q_{(u)}^{M}(\varphi, \varphi)-U_{0, u}^{M}(\varphi, \varphi)-u \sum_{\nu \text { in } M} m(y) \varphi^{2}(y)
$$

is well defined for $\varphi$ in $\boldsymbol{H}^{M}$ independent of $u>0$.

(iii) The Dirichlet space associated with $\left\{R^{M}{ }_{a}, a>0\right\}$ is $\left(H^{M}, Q^{M}\right)$.

Theorem 6.1 is a special case of the more general results proved in the first part of Section 5 in [17]. For the reader's convenience we repeat the proof here with appropriate simplifications.

Note first that for $u>0$

$$
R_{(u)}^{M} \varphi=G_{u} 1_{M} \varphi \quad \text { on } \quad M
$$

and therefore $R_{(u)}^{M}$ is bounded on $L^{2}(M, m)$. If $\varphi=R_{(u)}^{M} \psi$ with $\psi$ in $L^{2}(m)$ then also $\varphi=G_{u} 1_{M} \psi$ and therefore $H^{M}{ }_{u} \varphi$ belongs to $F$ and

$$
\begin{aligned}
E_{u}\left(H_{u}^{M} \varphi, H_{u}^{M} \varphi\right) & =E_{u}\left(G_{u} \psi 1_{M}, H_{u}^{M} \varphi\right) \\
& =\sum_{\nu \text { in } M} m(y) \varphi(y) \psi(y) .
\end{aligned}
$$

Such $\varphi$ are dense in the Dirichlet space associated with the $R_{(u) a}, a>0$ and it follows that this Dirichlet space is contained in $H^{M}$ and that $Q^{M}{ }_{(u)}$ is the associated Dirichlet norm. To complete the proof of (i) it suffices to show that all $\varphi$ in $H^{M}$ can be approximated by members of the associated Dirichlet space. But as $v \uparrow \infty$ we have $v G_{v} H^{M}{ }_{u} \varphi \rightarrow H^{M}{ }_{u \varphi}$ on $I$ and by the resolvent identity we can assume $\varphi=G_{u} g$ with $g$ in $L^{2}(I, m)$. But on $M$ we have $\varphi=H^{M}{ }_{u} \varphi=$ $H^{M}{ }_{u} G_{u} g=G_{u} \pi^{M}{ }_{u} g=R^{M}{ }_{(u)} \pi^{M}{ }_{u} g$ and (i) is proved.

(ii) follows from the calculation

$$
\begin{aligned}
Q_{(v)}^{M}(\varphi, \varphi)= & E_{v}\left(H_{v}^{M} \varphi, H_{v}^{M} \varphi\right) \\
= & E_{v}\left(H_{v}^{M} \varphi, H_{u}^{M} \varphi+(u-v) G_{v}^{D} H_{u}^{M} \varphi\right) \\
= & E_{v}\left(H_{v}^{M} \varphi, H_{u}^{M} \varphi\right) \\
= & E_{u}\left(H_{v}^{M} \varphi, H_{u}^{M} \varphi\right)+(v-u) \sum_{v} m(y) H_{v}^{M} \varphi(y) H_{u}^{M} \varphi(y) \\
= & E_{u}\left(H_{u}^{M} \varphi+(u-v) G_{u}^{D} H_{v}^{M} \varphi, H_{u}^{M} \varphi\right) \\
& +U_{u, v}^{M}(\varphi, \varphi)+(v-u) \sum_{v \text { in } M} m(y) \varphi^{2}(y) \\
= & Q_{(u)}^{M}(\varphi, \varphi)+U_{u, v}^{M}(\varphi, \varphi)+(v-u) \sum_{v \text { in } M} m(y) \varphi^{2}(y)
\end{aligned}
$$

where we have used the fact that $H^{M}{ }_{u}$ and $H^{M}{ }$ are projections onto the orthogonal complement of $F^{D}$ relative to $E_{u}$ and $E_{v}$. 
To prove (iii) note first that for $0<u<1$, for $a>0$ and for $\varphi, \psi$ in $L^{2}(M, m)$

$$
\begin{aligned}
& \sum_{\nu \text { in } M} m(y) \varphi(y) R_{(u) a}^{M} \psi(y) \\
= & \sum_{\nu \text { in } M} m(y) R_{(u) a}^{M} \varphi(y) \psi(y) \\
= & Q_{(1) a}\left(R_{(u) a}^{M} \varphi, R_{(1) a}^{M} \psi\right) \\
= & Q_{(u) a}^{M}\left(R_{(u) a}^{M} \varphi, R_{(1) a}^{M} \psi\right) \\
& +\sum_{\nu \text { in } M} m(y) R_{(u) a}^{M} \varphi(y)(1-u) \pi_{1}^{M} H_{u}^{M} R_{(1) a}^{M} \psi(y) \\
= & \sum_{\nu \text { in } M} m(y) \varphi(y)\left\{R_{(1) a}^{M} \psi(y)+(1-u) R_{(u) a}^{M} \pi_{1}^{M} H_{u}^{M} R_{(1) a}^{M} \psi(y)\right\}
\end{aligned}
$$

and it follows that

and similarly

$$
R_{(u) a}^{M}=R_{(1) a}^{M}+(1-u) R_{(u) a}^{M} \pi_{1}^{M} H_{u}^{M} R_{(1) a}^{M}
$$

$$
R_{(u) a}^{M}=R_{(1) a}^{M}+(1-u) R_{(1) a}^{M} \pi_{1}^{M} H_{u}^{M} R_{(u) a}^{M} .
$$

Passing to the limit $u \downarrow 0$ we get

$$
R_{(u) a}^{M}=R_{(1) a}^{M}\left\{1+\pi_{1}^{M} H_{0}^{M} R_{a}^{M}\right\} .
$$

If $\varphi=R^{M}{ }_{a} \psi$ with $\psi$ in $L^{2}(M, m)$, then also $\varphi=R^{M}{ }_{(1) a}\left\{\psi+\pi^{M}{ }_{1} H^{M}{ }_{0} \varphi\right\}$. Thus $\varphi$ belongs to $H$ and

$$
\begin{aligned}
& Q_{a}^{M}(\varphi, \varphi)=Q_{(1) a}^{M}(\varphi, \varphi)-\sum_{\nu \text { in } M} m(y) \varphi(y) \pi_{1}^{M} H_{0}^{M} \varphi(y) \\
& =\sum_{\nu \text { in } M} m(y) \varphi(y)\left\{\psi(y)+\pi_{1}^{M} H_{0}^{M} \varphi(y)\right\} \\
& \quad-\sum_{\nu \text { in } M} m(y) \varphi(y) \pi_{1}^{M} H_{0}^{M} \varphi(y) \\
& =\sum_{\nu \text { in } M} m(y) \varphi(y) \psi(y) .
\end{aligned}
$$

Again it follows that the Dirichlet space associated with $R^{M}{ }_{a}, a>0$ is contained in $H^{M}$ and that $Q^{M}$ is the associated Dirichlet norm. But it follows from (6.9) and from Theorem 4.1-(ii) that the Dirichlet space associated with $R_{a}^{M}, a>0$ contains the Dirichlet space associated with $R_{(1) a}^{M}, a>0$ and (iii) is completely prove.

The crucial result for classification is

Theorem 6.2. $F$ is contained in $\mathcal{F}$ and $Q^{M}-\frac{1}{2} \Theta^{M}\langle$,$\rangle is contractive on H^{M}$.

The meaning of Theorem 6.2 is this. If $\varphi$ belongs to $H^{M}$ and if $\psi$ is a normalized contraction of $\varphi$, then

$$
Q^{M}(\varphi, \varphi)-\frac{1}{2} \Theta^{M}\langle\varphi, \varphi\rangle \geqq Q^{M}(\psi, \psi)-\frac{1}{2} \Theta^{M}\langle\psi, \psi\rangle .
$$

(See (4.3) for the definition of normalized contraction.) 
Theorem 6.2 is a special case of Theorem 5.8 in [17]. Again for the reader's convenience we repeat the proof here with simplifications.

It suffices to consider $\varphi$ in $H^{M}$ bounded and $\psi$ a normalized contraction of $\varphi$. For $0<u<v$ and for $a>0$,

$$
\begin{aligned}
a \sum_{y \text { in } M} m(y)\{\varphi- & \left.a R_{(v) a}^{M} \varphi\right\}(y) \varphi(y)-a \sum_{y \text { in } M} m(y)\left\{\psi-a R_{(v) a}^{M} \psi\right\}(y) \psi(y) \\
= & a \sum_{v \text { in } M} m(y)\left\{1-a R_{(v) a}^{M} 1\right\}(y)\left\{\varphi^{2}-\psi^{2}\right\}(y) \\
& +\frac{1}{2} a \sum_{\nu, z \text { in } M} m(y) a R_{(v) a}^{M}(y, z)\left(\{\varphi(y)-\varphi(z)\}^{2}-\{\psi(y)-\psi(z)\}^{2}\right) \\
\geq & a \sum_{y \text { in } M} m(y)\left\{1-a R_{(v) a}^{M} 1\right\}(y)\left\{\varphi^{2}-\psi^{2}\right\}(y) \\
= & a \sum_{\nu \text { in } M} m(y)\left\{1-a R_{(u) a}^{M} 1\right\}(y)\left\{\varphi^{2}-\psi^{2}\right\}(y) \\
& +a^{2} \sum_{\nu \text { in } M} m(y)(v-u) \pi_{v}^{M} H_{u}^{M} R_{(v) a}^{M} 1(y) R_{(u) a}^{M}\left\{\varphi^{2}-\psi^{2}\right\}(y) \\
\geq & a^{2} \sum_{\nu \text { in } M} m(y)(v-u) \pi_{v}^{M} H_{u}^{M} R_{(u) a}^{M} 1(y) R_{(u) a}^{M}\left\{\varphi^{2}-\psi^{2}\right\}(y) .
\end{aligned}
$$

As $a \uparrow \infty$ the initial expression converges to

$$
Q_{(v)}^{M}(\varphi, \varphi)-Q_{(v)}^{M}(\psi, \psi)
$$

by Theorem 4.1. On the other hand $a R^{M}{ }_{a} 1 \uparrow 1$ and for a subsequence $a R_{(u) a}^{M}\left\{\varphi^{2}-\psi^{2}\right\} \rightarrow \varphi^{2}-\psi^{2}$ and so by Fatou's lemma

or equivalently

$$
Q_{(v)}^{M}(\varphi, \varphi)-Q_{(v)}^{M}(\psi, \psi) \geq(v-u) \sum_{\nu \text { in } M} m(y) \pi_{v}^{M} H_{u}^{M}\left\{\varphi^{2}-\psi^{2}\right\}(y)
$$

$$
Q_{(u)}^{M}(\varphi, \varphi)-Q_{(u)}^{M}(\psi, \psi) \geq U_{u, v}^{M}\langle\varphi, \varphi\rangle-U_{u, v}^{M}\langle\psi, \psi\rangle .
$$

The theorem follows upon letting $v \uparrow \infty$, then $u \downarrow 0$ and applying (5.24).

Remark. The heuristic argument behind Theorem 6.2 is this. The process corresponding to $Q^{M}{ }_{(v)}$ is obtained from the process corresponding to $Q^{M}{ }_{(u)}$ by replacing "jumps at the rate $U^{M}{ }_{u, v}(y, z) m(z)$ " by "killing at the rate $(v-u) \pi^{M}{ }_{v} H^{M}{ }_{u} 1(y) . "$ Therefore $Q^{M}{ }_{(u)}$ must already contain "jumping at the rate $U^{M}{ }_{u, v}(y, z) m(z)$." That is, (6.10) must be valid.

If $f$ belongs to $F$ then also $f$ belongs to $F$ and therefore by Theorem 2.11 and Theorem 3.4, $f$ has a unique representation

$$
f=H h^{\cdot}+g
$$

with $h$ in $\mathfrak{F}$ and $g$ in $\mathscr{F}^{\mathrm{min}}$. Since the operators $H^{M}{ }_{u}$ form a decreasing family of orthogonal projections relative to $E_{u}$ on $F$ as $D$ increases and since $H^{M}{ }_{u} g \rightarrow 0$ and $H^{M}{ }_{u} H h^{*} \rightarrow H_{u} h^{r}$, it follows that for $u>0$ also $f$ has a unique representation

$$
f=H_{u} h+g_{u}
$$


with $g_{u}$ in $F^{\text {min }}$. But then $H h^{\cdot}-H_{u} h$ belongs to $\mathcal{F}^{\text {min }}$ and therefore $h$ vanishes [a.e. $\ell^{1}$ ] on the passive boundary $\Delta_{0}-\Delta_{m}$. Define

$$
H=\left\{h^{\cdot} \text { in } \mathfrak{F}: H_{1} h^{\cdot} \text { is in } F\right\} .
$$

By (5.32) and Theorem 4.3 also $H_{u} h$ is in $F$ for $u>0$ and $h$ in $H$. Thus for $u>0$

$$
Q_{(u)}\left(h^{\cdot}, h^{\cdot}\right)=E_{u}\left(H_{u} h^{\cdot}, H_{u} h^{*}\right)
$$

is well defined for $h$ in $\boldsymbol{H}$. Arguing as in the proof of (6.7), we get

$$
Q_{(v)}\left(h^{\cdot}, h^{*}\right)=Q_{(u)}\left(h^{*}, h^{*}\right)+U_{u, v}\left(h^{*}, h^{*}\right)
$$

for $0<u<v$ and therefore

$$
U_{0, u}\left(h^{\cdot}, h^{\cdot}\right)<+\infty
$$

and also

$$
Q\left(h^{\cdot}, h^{\cdot}\right)=Q_{(u)}\left(h^{\cdot}, h^{\cdot}\right)-U_{0, u}\left(h^{\cdot}, h^{\cdot}\right)
$$

is well defined independent of $u>0$ for $h$ in $H$. For $D$ finite,

and therefore by Theorem 6.2

$$
\begin{aligned}
Q_{(u)}\left(h^{\cdot}, h^{*}\right) & =E_{u}\left(H_{u} h^{\cdot}, H_{u} h^{\cdot}\right) \\
& =E_{u}\left(H_{u}^{M} H_{u} h^{\cdot}, H_{u}^{M} H_{u} h^{\circ}\right) \\
& =Q_{(u)}^{M}\left(H_{u} h^{\cdot}, H_{u} h^{\circ}\right)
\end{aligned}
$$

$$
\begin{aligned}
Q_{(u)}\left(h^{\cdot}, h^{*}\right) & -\frac{1}{2} \Theta^{M}\left(H_{u} h^{\cdot}, H_{u} h^{*}\right)-U_{0, u}^{M}\left(h^{\cdot}, h^{*}\right) \\
& -\sum_{x \text { in } M} m(x)\left\{H_{u} h^{\cdot}\right\}^{2}(x)
\end{aligned}
$$

is contractive on $H$. By Theorem 2.11

converges to

$$
\frac{1}{2} \Theta^{M}\left\langle H_{u} h^{\cdot}, H_{u} h^{\cdot}\right\rangle=\mathcal{E}\left(H^{M} H_{u} h^{\cdot}, H^{M} H_{u} h^{\cdot}\right)
$$

$$
\varepsilon\left(H H_{u} h^{\cdot}, H H_{u} h^{\cdot}\right)=\frac{1}{2} \alpha(0) \Theta\left\langle h^{\cdot}, h^{\cdot}\right\rangle
$$

as $D$ increases to $I$. It is easy to check directly that

$$
U_{0, u}^{M}\left(H_{u} h^{\cdot}, H_{u} h^{\circ}\right)+\sum_{x \text { in } M} m(x)\left\{H_{u} h^{\cdot}\right\}^{2}(x)
$$

converges to $U_{0, u}\left(h^{*}, h^{*}\right)$ and we conclude that

is contractive on $\boldsymbol{H}$. Also,

$$
Q\left(h^{\cdot}, h^{\cdot}\right)-\frac{1}{2} \alpha(0) \Theta\left\langle h^{\cdot}, h^{\cdot}\right\rangle
$$

$$
\begin{aligned}
U_{0,1}\left(h^{*}, h^{\cdot}\right)+\frac{1}{2} \alpha(0) \Theta\left\langle h^{*}, h^{\cdot}\right\rangle \geqq & U_{0,1}\left(h^{\cdot}, h^{\cdot}\right)+\frac{1}{2} U_{0,1}\left\langle h^{\cdot}, h^{\cdot}\right\rangle \\
& +\int \pi_{1} m(d y) \pi_{1} p(y) h^{\cdot 2}(y) \\
= & U_{0,1}\left(h^{\cdot 2}, 1\right)+\sum_{x} m(x) p(x) H_{1} h^{\cdot 2}(x)
\end{aligned}
$$




$$
\begin{aligned}
& =\sum_{x} m(x)\left\{H_{0} 1_{\Delta_{m}}(x)+p(x)\right\} H_{1} h^{\cdot 2}(x) \\
& =\int \pi_{1} m(d y) h^{\cdot 2}(y)
\end{aligned}
$$

Thus $H$ is a linear subset of $L^{2}\left(\Delta_{m}, \pi_{1} m\right)$ and it follows that actually $(H, Q)$ is a Dirichlet space relative to $L^{2}\left(\Delta_{m}, \pi_{1} m\right)$. We have proved the direct part of the following theorem.

Theorem 6.3. Let $\boldsymbol{P}_{t}(x, y)$ be a standard transition matrix satisfying (4.8), $(4,10)$ and $(4.12)$ and let $(F, E)$ be the associated Dirichlet space. Then there is a unique Dirichlet space $(H, Q)$ on $L^{2}\left(\Delta_{m}, \pi_{1} m\right)$ satisfying

6.3.1. $H$ is contained in $\mathcal{H}$ and $Q\left(h^{\cdot}, h^{*}\right)-\frac{1}{2} \alpha(0) \Theta\left\langle h^{\cdot}, h^{\circ}\right\rangle$ is contractive on $H$,

6.3.2. $h^{\cdot}$ belongs to $H$ if and only if $H_{1} h^{\cdot}$ belongs to $F$ and then $Q\left(h^{*}, h^{*}\right)=$ $E_{1}\left(H_{1} h^{\cdot}, H_{1} h^{\cdot}\right)-U_{0,1}\left(h^{\cdot}, h^{\cdot}\right)$.

Conversely if $(H, Q)$ is a Dirichlet space on $L^{2}\left(\Delta_{m}, \pi_{1} m\right)$ satisfying 6.3 .1 , then there is a unique standard transition matrix satisfying (4.8), (4.10) and (4.12) with associated Dirichlet space $(F, E)$ such that 6.3 .2 is satisfied.

The subscript 1 can be stripped away from 6.3 .2 by introducing the extended Dirichlet space $F_{(e)}$ as in Section 8 of [17]. Also it can be shown using the techniques of [17] that the Dirichlet space $(H, Q)$ is associated with a time changed process on the boundary $\boldsymbol{X}-\boldsymbol{I}$.

To prove the converse, fix a Dirichlet space $(H, Q)$ on $L^{2}\left(\Delta_{m}, \pi_{1} m\right)$ satisfying 6.3.1. For $u>0$ define $Q_{(u)}$ on $H$ by (6.12). (Notice that $U_{0, u}(\varphi, \varphi)$ converges for any $\varphi$ in $L^{2}\left(\Delta_{m}, \pi_{1} m\right)$.) We can write

$$
\begin{aligned}
& Q_{(u)}\left(h^{\cdot}, h^{\prime}\right)=Q\left(h^{\cdot}, h^{\cdot}\right)-\frac{1}{2} U_{0, u}\left\langle h^{\cdot}, h^{\cdot}\right\rangle+U_{0, u}\left(h^{\cdot 2}, 1\right) \\
&= Q\left(h^{\cdot}, h^{\cdot}\right)-\frac{1}{2} \alpha(0) \Theta\left\langle h^{\cdot}, h^{\cdot}\right\rangle+U_{0, u}\left(h^{\cdot 2}, 1\right) \\
&+\int \pi_{1} m(d y) u \pi_{u} p(y) h^{\cdot 2}(y) \\
&+\left\{\frac{1}{2} \alpha(0) \Theta\left\langle h^{\cdot}, h^{\cdot}\right\rangle-\frac{1}{2} U_{0, u}\left\langle h^{\cdot}, h^{\cdot}\right\rangle-\int \pi_{1} m(d y) u \pi_{u} p(y) h^{\cdot 2}(y)\right\}
\end{aligned}
$$

and it follows that

$$
Q_{(u)}\left(h^{\circ}, h^{\cdot}\right)-\int \pi_{1} m(d y) u \pi_{u} \mathbf{P}(y) h^{\cdot 2}(y)
$$

is contractive on $H$. This together with the trivial estimate

$$
U_{0,1}\left(h^{.2}, 1\right) \leqq \max (1,1 / u) U_{0, u}\left(h^{.2}, 1\right)
$$


and the calculation (6.15) guarantees that $Q_{(u)}$ dominates a multiple of the standard inner product on $L^{2}\left(\Delta_{m}, \pi_{1} m\right)$ and therefore $H$ is a Hilbert space relative to the inner product $Q_{(u)}$. Let $R_{(u)}$ be the unique operator mapping $L^{2}\left(\Delta_{m}, \pi_{1} m\right)$ into $H$ such that

$$
Q_{(u)}\left(R_{(u)} \varphi, h^{*}\right)=\int \pi_{1} m(d y) \varphi(y) h^{\cdot}(y)
$$

for $\varphi$ in $L^{2}\left(\Delta_{m}, \pi_{1} m\right)$ and for $h$ in $H$. The desired resolvent operators $G_{u}, u>0$ are defined on $L^{2}(m)$ by

$$
G_{u} f=G_{u}^{\mathrm{min}} f+H_{u} R_{(u)} \pi_{u} f
$$

and it follows that also

$$
G_{u} f=G_{u}^{\mathrm{min}} f+H_{u} G_{u} f
$$

with $H_{u}$ regarded as an operator on $F$. It is easy to check that each $G_{u}$ is a bounded symmetric operator on $L^{2}(m)$. To establish the resolvent identity we argue as in the proof of Lemma 6.3 in [17]. For $0<u<v$ and for $\varphi$ in $L^{2}\left(\Delta_{m}, \pi_{1} m\right)$

$$
\begin{aligned}
\int \pi_{1} m(d y) \varphi(y) R_{(u)} \varphi(y) & =Q_{(v)}\left(R_{(v)} \varphi, R_{(u)} \varphi\right) \\
& =Q_{(u)}\left(R_{(v)} \varphi, R_{(u)} \varphi\right)+U_{0, u}\left(R_{(v)} \varphi, R_{(u)} \varphi\right) \\
& =\int \pi_{1} m(d y) \varphi(y)\left\{R_{(v)} \varphi+R_{(u)} U_{u, v} R_{(v)} \varphi\right\}
\end{aligned}
$$

and therefore

$$
R_{(u)}=R_{(v)}+(v-u) R_{(u)} \pi_{u} H_{v} R_{(v)}
$$

But then

$$
\begin{aligned}
G_{u}-G_{v}= & G_{u}^{\mathrm{min}}-G_{v}^{\mathrm{min}}+H_{u} R_{(u)} \pi_{u}-H_{v} R_{(v)} \pi_{v} \\
= & (v-u) G_{u}^{\mathrm{min}} G_{v}^{\mathrm{min}}+H_{u} R_{(u)} \pi_{v}\left\{1+(v-u) G_{u}^{\mathrm{min}}\right\} \\
& -\left\{1-(v-u) G_{v}^{\mathrm{min}}\right\} H_{u} R_{(v)} \pi_{v} \\
= & (v-u) G_{u}^{\mathrm{min}} G_{v}^{\mathrm{min}}+(v-u) H_{u} R_{(u)} \pi_{u} H_{v} R_{(v)} \pi_{v} \\
& +(v-u) H_{u} R_{(u), \pi_{u}} G_{v}^{\mathrm{min}}+(v-u) G_{u}^{\mathrm{min}} H_{v} R_{(v)} \pi_{(v)} \\
= & (v-u) G_{u} G_{v}
\end{aligned}
$$

and the resolvent identity is established. To show that the $G_{u}$ are submarkovian let $q \geqq 0$ be any bounded function dominated by $u \pi_{u} 1$ and note that for $h$ in $H$ and $\varphi$ in $L^{2}\left(\Delta_{m}, \pi_{1} m\right)$ 


$$
\begin{aligned}
Q_{(u)}\left(h^{\cdot}-R_{(u)} q \varphi, h^{\cdot}-\right. & \left.R_{(u)} q \varphi\right)=Q_{(u)}\left(h^{\cdot}, h^{\cdot}\right)-\int \pi_{1} m(d y) q(y) h^{\cdot 2}(y) \\
& +\int \pi_{1} m(d y) q(y)\left\{h^{\cdot 2}(y)+\varphi(y) R_{(u)} q \varphi(y)-2 h^{\cdot}(y) \varphi(y)\right\} \\
= & Q_{(u)}\left(h^{\cdot}, h^{\cdot}\right)-\int \pi_{1} m(d y) q(y) h^{\cdot 2}(y) \\
& +\int \pi_{1} m(d y) q(y)\left\{h^{\cdot}-\varphi\right\}^{2}(y) \\
& +\int \pi_{1} m(d y) q(y)\left\{\varphi^{2}(y)-\varphi(y) R_{(u)} q \varphi(y)\right\}
\end{aligned}
$$

and therefore the functional

$$
\Phi\left(h^{*}\right)=Q_{(u)}\left(h^{\cdot}, h^{*}\right)-\int \pi_{1} m(d y) q(y) h^{\cdot 2}(y)+\int \pi_{1} m(d y) q(y)\left\{h^{\cdot}-\varphi\right\}^{2}(y)
$$

has the unique minimum $h^{\cdot}=R_{(u)} q \varphi$. From the contractivity of (6.16) it follows that $\Phi\left(T h^{\circ}\right) \leqq \Phi\left(h^{\circ}\right)$ whenever $T$ satisfies $\left(4.7^{\prime}\right)$. Reasoning as in the first part of Section 4 we conclude that $R_{(u)}$ is a nonnegative operator and that $R_{(u)} q \leqq 1$. Letting $q$ increase to $u \pi_{u} 1$ we conclude that $u R_{(u)} \pi_{u} 1 \leqq 1$ and therefore $u G_{u} 1 \leqq 1$. Thus the $G_{u}$ are submarkovian and we have proved that the $G_{u}$ form a symmetric submarkovian resolvent on $L^{2}(m)$. Let $(F, E)$ be the associated Dirichlet space.

To analyze $(F, E)$ we show first that for $g \geqq 0$ in $L^{2}(m)$

$$
v G_{u+v} H_{u} G_{u} g \uparrow H_{u} G_{u} g
$$

on $I$ as $v \uparrow \infty$. The calculation

$$
\begin{aligned}
v G_{u+v} H_{u} G_{u} g & =v G_{u+v}^{\min } H_{u} G_{u} g+v H_{u+v} G_{u+v} H_{u} G_{u} g \\
& =H_{u} G_{u} g-H_{u+v} G_{u} g+v H_{u+v} G_{u+v} H_{u} G_{u} g \\
& \leqq H_{u} G_{u} g-H_{u+v} G_{u} g+v H_{u+v} G_{u+v} G_{u} g \\
\leqq & H_{u} G_{u} g-H_{u+v} G_{u} g+H_{u+v} G_{u} g
\end{aligned}
$$

shows that

$$
v G_{u+v} H_{u} G_{u} g \leqq H_{u} G_{u} g
$$

and then the relation

$$
v G_{u+v} H_{u} G_{u} g=w G_{u+w}\left\{(v / w)+[1-(v w)] v G_{u+v}\right\} H_{u} G_{u} g
$$

shows that

$$
v G_{u+v} H_{u} G_{u} g \leqq w G_{u+w} H_{u} G_{u} g \leqq H_{u} G_{u} g
$$


for $0<v<w$. Finally convergence in (6.19) follows from

$$
\begin{aligned}
\sum_{x} m(x) v G_{u+v} H_{u} G_{u} g(x) f(x) & =\sum_{x} m(x) g(x) v H_{u} G_{u} G_{u+v} f(x) \\
& =\sum_{x} m(x) g(x)\left\{H_{u} G_{u} f-H_{u} G_{u+v} f\right\}(x)
\end{aligned}
$$

since it suffices to consider $g$ integrable and since $H_{u} G_{u+v} f \rightarrow 0$ boundedly for $f$ bounded as $v \uparrow \infty$. Next we have

$$
\operatorname{Lim} v \sum_{x} m(x)\left\{1-v G_{u+v}\right\} H_{u} G_{u} g(x) G_{u}^{\text {min }} f(x)=0
$$

with the limit taken as $v \uparrow \infty$ for $f, g$ in $L^{2}(m)$. This follows from (6.19) and the calculation

$$
\begin{array}{rl}
v \sum_{x} m(x)\left\{1-v G_{u+v}\right\} H_{u} G_{u} g(x) G_{u}^{\mathrm{min}} & f(x) \\
= & v \sum_{x} m(x)\left\{H_{u} G_{u} g-v G_{u+v}^{\mathrm{min}} H_{u} G_{u} g\right\}(x) G_{u}^{\mathrm{min}} f(x) \\
& -v^{2} \sum_{x} m(x) H_{u+v} G_{u+v} H_{u} G_{u} g(x) G_{u}^{\mathrm{min}} f(x) \\
= & v \sum_{x} m(x) G_{u+v}^{\mathrm{min}} H_{u} G_{u} g(x) f(x) \\
& -v^{2} \sum_{x} m(x) G_{u+v}^{\mathrm{min}} H_{u} G_{u+v} H_{u} G_{u} g(x) f(x)
\end{array}
$$

since it suffices to consider $f, g \geqq 0$. From (6.20) it follows by Theorem 4.1-(ii) that both $H_{u} G_{u} f$ and $G_{u}^{\min } f$ belong to $F$ for $f$ in $L^{2}(m)$ and for $u>0$ and that

$$
\begin{aligned}
& E_{u}\left(G_{u}^{\mathrm{min}} f, G_{u}^{\mathrm{min}} g\right)=E_{u}\left(G_{u}^{\mathrm{min}} f, G_{u}^{\mathrm{min}} g\right) \\
& E_{u}\left(G_{u}^{\mathrm{min}} f, H_{u} G_{u} g\right)=0
\end{aligned}
$$

for $f, g$ in $L^{2}(m)$. It follows from (6.21) that $(F, E)$ satisfies (4.15) and therefore the corresponding transition matrix satisfies (4.8), (4.10) and (4.12). Finally from (6.22) we have for $f, g$ in $L^{2}(m)$

$$
\begin{aligned}
E_{1}\left(H_{1} G_{1} f, H_{1} G_{1} g\right) & =E_{1}\left(G_{1} f, H_{1} G_{1} g\right) \\
& =\sum_{x} m(x) f(x) H_{1} R_{(1)} \pi_{1} g \\
& =Q_{(1)}\left(R_{(1)} \pi_{1} f, R_{(1)} \pi_{1} g\right)
\end{aligned}
$$

and 6.3.2 follows since the range of $H_{1} G_{1}$ is dense in $H$. This completes the proof of Theorem 6.3.

\section{References}

1. A. Beurling \& J. Deny, Dirichlet spaces, Proc. Nat. Acad. Sci. U.S.A. 45 (1959), 208-215.

2. K. L. Chung, Markov chains with stationary transition probabilities, second Edition, Springer-Verlag, New York, 1967.

3. $\longrightarrow$ Lectures on boundary theory for Markov chains, Annals of Math. Studies No. 65, Princeton University Press, Princeton, 1970. 
4. - On the boundary theory for Markov chains, Acta Math. 110 (1963), 19-77; Acta Math. 115 (1966), 11-163.

5. J. L. Dоoв, Boundary properties of functions with finite Dirichlet integrals, Ann. Inst. Fourier (Grenoble) 13 (1962), 573-621.

6. J. Elloto, Dirichlet spaces and boundary conditions for submarkovian resolvents, J. Math. Anal. Appl. 36 (1971), 251-282.

7. W. Feller, On boundaries and lateral conditions for the Kolmogorov differential equations, Ann. of Math. 65 (1967), 527-570.

8. M. Fukishima, On Feller's kernel and the Dirichlet norm, Nagoya Math. J. 24 (1964), 167175.

9. - A construction of reflecting barrier Brownian motions for bounded domains, Osaka J. Math. 4 (1967), 183-215.

10. - On boundary conditions for multi-dimensional Brownian motion with symmetric resolvents, J. Math. Soc. Japan 21 (1969), 485-526.

11. G. A. Hunt, Markov chains and Martin boundaries, Ill. J. Math. 4 (1960), 313-340.

12. J. G. Kemeny, A. W. Knapp \& J. L. Snell, Denumerable Markov chains, Van Nostrand, New York, 1966.

13. L. NaÏм, Sur le role de la frontiere de R. S. Martin dans la theorie du potentiel, Annales Inst. Fourier 7 (1957), 183-281.

14. D. Ornstein, The differentiability of transition functions, Bull. Amer. Math. Soc. 66 (1960), 36-39.

15. K. R. Parthasarathy, Probability measures on metric spaces, Academic Press, New York, 1967.

16. T. Shiga \& T. Watanabe, On Markov chains similar to the reflecting barrier Brownian motion, Osaka J. Math. 5 (1968), 1-33.

17. M. L. Silverstein, Dirichlet spaces and random time change, Ill. J. of Math. 17 (1973), 1-72.

18. - The reflected Dirichlet space, (to appear in the Ill. J. of Math.)

19. M. WeIL, Quasi-processus, Seminaire de Probabilitiés IV, Springer-Verlag Lect. Notes in Math. 124, 1970.

Added in proof.

20. M. L. Silverstein, Symmetric Markov processes, (monograph in preparation).

21. - Symmetric Markov chains, (to appear in the Annals of Prob.).

This research was partially supported by National Science Foundation Grant 24239.

University of Southern California

Date communicated: August 24, 1973 